\title{
Changes in innervation of lumbar motoneurons and organization of premotor network following training of transected adult rats
}

\author{
Loubna Khalki ${ }^{\mathrm{a}, \mathrm{b}}$, Karina Sadlaoud ${ }^{\mathrm{a}}$, Julie Lerond ${ }^{\mathrm{a}}$, Jacques-Olivier Coq ${ }^{\mathrm{a}}$, Jean-Michel Brezun ${ }^{\mathrm{c}}$, \\ Laurent Vinay ${ }^{\mathrm{a}}$, Patrice Coulon ${ }^{\mathrm{a}}$, Hélène Bras ${ }^{\mathrm{a}, *}$ \\ a Institut de Neurosciences de la Timone, UMR 7289, Team P3M, CNRS and Aix Marseille Université, Marseille, France \\ b Neuroscience Laboratory, Faculty of Medicine, Mohammed VI University of Health Sciences, Casablanca, Morocco

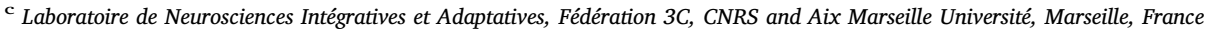

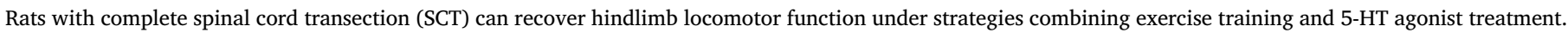

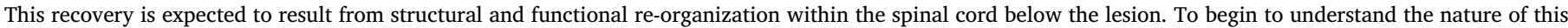

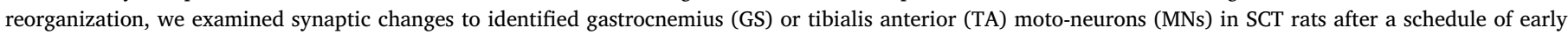

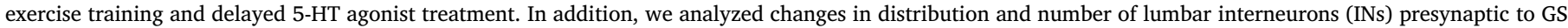
MNs using retrograde transneuronal transport of rabies virus.

In SCT-untrained rats, we found few changes in the density and size of inhibitory and excitatory inputs impinging on cell bodies of TA and GS MNs compared to

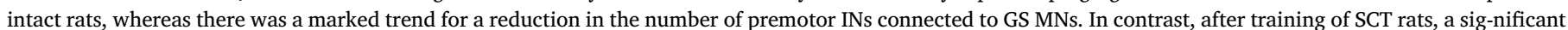

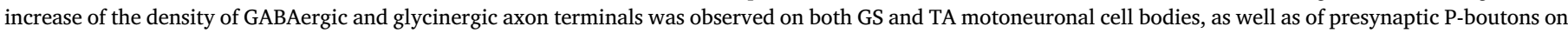

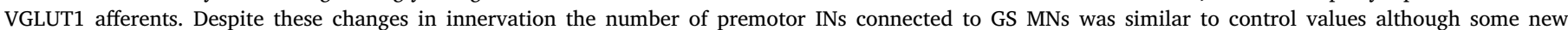

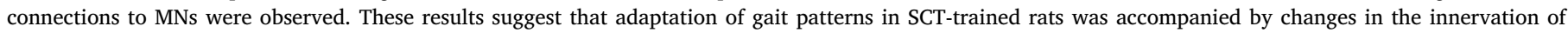
lumbar MNs while the distribution of the spinal premotor circuitry was relatively preserved.

\section{Introduction}

Every year, 250,000 traumatic spinal cord injuries are reported worldwide, mainly caused by accidents in the daily life. They constitute a major cause of disability, with $53 \%$ of the patients suffering from lower limb paresis. Animals with complete spinal cord transection (SCT) are highly relevant models of paraplegia to study the induced plasticity of the sensorimotor networks caudal to the lesion (Rossignol, 2006). After SCT, the loss of supraspinal descending fibers, in particular serotonergic afferents, creates a depressed state in the sub-lesional networks. These networks are unable to interact dynamically with afferent sensory inputs, depriving motoneurons (MNs) of the normal rhythmic alternating patterns of excitation and inhibition (Rossignol and Frigon, 2011). Exercise training is an essential component of neurologic rehabilitation programs in patients with spinal cord injury (Harkema et al., 2012). In animals with complete SCT, a wide range of exercise training protocols has been used to improve locomotor function (Battistuzzo et al., 2012; Roy et al., 2012). They include treadmill training with robotic or manual assistance (Alluin et al., 2015; de Leon and Acosta, 2006; Fong et al., 2005; Moshonkina et al., 2004; Timoszyk et al., 2005), body weight support treadmill (Cantoria et al., 2011; Cha et al., 2007; Ichiyama et al., 2011; Timoszyk et al., 2005; Zhang et al., 2007), cycling (Cote et al., 2014; Nothias et al., 2005) and voluntary exercises in environmentally enriched housing (Burke et al., 2007; Lankhorst et al., 2001; van Meeteren et al., 2003). The concept of taskdependent plasticity has been clearly established from these experiments, emphasizing that repetitive activation of appropriate sensory and motor pathways using task-specific training reinforces the activity of a unique combination of neuronal networks in an activity-dependent manner (de Leon et al., 1998; Edgerton et al., 2008; Musienko et al., 2011).

Nevertheless, in small species such as rats and mice the level of

\footnotetext{
* Corresponding author at: Institut de Neurosciences de la Timone (INT), UMR 7289 CNRS-Aix Marseille Université, Campus Santé Timone, 27 Bd Jean Moulin, 13385 Marseille Cedex 05, France.

E-mail address: helene.bras@univ-amu.fr (H. Bras).
} 
locomotor recovery after SCT remains low even after exercise (Fong et al., 2005). However, combining treadmill training with serotonin agonist treatment has been shown to generate significant recovery of hindlimb locomotor function in SCT rodent as shown in several studies (Antri et al., 2002; Dugan and Shumsky, 2015; Feraboli-Lohnherr et al., 1999; Fong et al., 2005; Guertin, 2004; Orsal et al., 2002; Slawinska et al., 2012). These studies, moreover, revealed some interesting features of this combined treatment: first, treatment with serotonergic agonists does not directly generate stepping but facilitates stepping only when triggered by exercise training (Fong et al., 2005). Second, chronic long-term treatment of SCT rats with $5-\mathrm{HT}_{2}$ agonists starting shortly after the lesion had only limited effect on body weight bearing (Antri et al., 2002). With this in mind, we adapted a training protocol of SCT rats based on two hypotheses: 1) combining different exercise training tasks should activate several sub-lesional neuronal spinal networks in SCT rats; 2) treatment with 5HT agonist should follow several weeks of initial exercise training alone in order to facilitate optimal recovery of stepping. The overground stepping abilities of the SCT-trained rats were tested qualitatively and quantitatively by means of BBB scoring (Basso et al., 1995) and Catwalk analysis (Cote et al., 2012; Hamers et al., 2001).

Earlier studies on the effects of training on the associated neurochemical plasticity in the sublesional spinal cord focused mainly on inhibition, including levels of glutamate decarboxylase (GAD67) in adult cats (Tillakaratne et al., 2010; Tillakaratne et al., 2000), expression of GABAA $\gamma 2$ receptor subunit in lumbar MNs of neonatal SCT rats (Khristy et al., 2009), as well as expression levels of potassium-chloride co-transporter KCC2 in adult rats (Cote et al., 2014). The effects of transection and subsequent training on the excitation/inhibition balance, which is a fundamental feature of in vivo networks, remain unclear. In a neonatal model of SCT, locomotor training maintain the control levels of inhibitory to excitatory boutons on lumbar MNs (Ichiyama et al., 2011), whereas other studies showed that there is no significant differences in the densities of glutamatergic (VGLUT1) and glycinergic (GlyT2) inputs between trained and untrained animals (Cantoria et al., 2011). Therefore, the first objective of the present study was to analyze the training-induced re-organization of inhibitory (GABAergic and glycinergic) and excitatory (glutamatergic, VGLUT1) inputs onto the somata of identified ankle flexor tibialis anterior (TA) and ankle extensor gastrocnemius (GS) MNs using quantitative immunohistochemistry. We also examined the pre-synaptic inhibitory Pboutons apposed on VGLUT1 afferents.

Structural changes in the neuronal premotor circuitry upstream of the lumbar MNs in SCT-untrained and in SCT-trained animals have never been investigated. In particular, no information is available on the level of reorganization of premotor INs connected to a specific pool of MNs following SCT and after locomotor training of SCT animals. Therefore, the second aim of the present study was to investigate the potential re-organization of premotor interneurons (INs) connected to GS MNs in SCT-untrained and SCT-trained adult rats by means of retrograde transneuronal tracing with rabies virus (RABV).

\section{Methods}

\subsection{Animals}

The experiments were performed on 37 adult Wistar female rats (Charles River, France). 28 of them received a complete spinal cord transection (SCT) at the T8-T9 level. All procedures were approved by the ethics committee in Neurosciences at the Institut de Neurosciences de la Timone, INT-Marseille (CEEA $N^{\circ} 71$, authorizations $n^{\circ} 03225.01$ and $\mathrm{n}^{\circ}$ 02167.01). The number of animals used for the different experiments is given in Fig. 1A.

\subsection{Lesion surgery}

Rats (220-280 g) were anaesthetized with i.p. injections of a mixture of ketamine (Imalgene, Merial, $50 \mathrm{mg} / \mathrm{kg}$ ) and dormitor (Médétomidine, Janssen $0.25 \mathrm{mg} / \mathrm{kg}$ ). After laminectomy, the spinal cord was transected at the thoracic level (T8-T9). After SCT, the animals received $5 \mathrm{~mL}$ of $\mathrm{NaCl} \mathrm{s}$-c. Food was placed in the cage and the rats were kept warm until awakening. Buprenorphine was administered (Vetergesic, Sogeva, $0.05 \mathrm{mg} / \mathrm{kg}$, s-c.) to the rats twice in $24 \mathrm{~h}$. Twice a day, their bladder was manually emptied until recovery of urinary function, their temperature and hydration were checked and any clinical sign of pain was observed. Surgery and post-operatory cares and nursing were performed by NSrepair, Inc., INT, Marseille. One week post-lesion, the animals to be trained were housed 3 per cage on a 12hour light/dark cycle with access to food and water ad libitum.

\subsection{Training procedure}

The plurisensorimotor procedure used in this study required standardization of the different stages of exercise training and analyses. The training, performed on 14 SCT rats, started 7 days after transection and was performed every day during 10 weeks. The procedure combined free moving in enriched environment, imposed locomotion in a treadmill carrousel and exercises of physiotherapy (Video 1). Environmentally enriched housing conditions ( 4 to $6 \mathrm{~h} /$ day) were set within ranges defined in previous studies (Burke et al., 2007; Lankhorst et al., 2001; van Meeteren et al., 2003), with groups of 3 to 6 animals kept in a clear cage $(120 \times 30 \times 34 \mathrm{~cm})$ in which the position of objects (tubes, bilateral staircase, cardloard box) and floor texture were regularly changed to motivate the rats to spontaneous exploration. Imposed locomotion $(2 \times 10 \mathrm{~min} /$ day $)$ was performed in a treadmill carrousel adapted from Martinez and collaborators (Martinez et al., 2009), designed as 3 individual boxes hanging over a rotating plateau in which the animals adapted their moving rate to the rotation speed of the plateau (Video 1; linear speed $3 \mathrm{~m} / \mathrm{min}$ ). This speed was a compromise to avoid tiredness of the SCT rats (paraplegic after transection) and to insure secured simultaneous training of the three rats. The training also included exercises of physiotherapy, imposing alternating flexion and extension of both hindlimbs (100 movements, twice a day). After training, the rats were housed in groups of 3 (as well as intact and SCT-untrained animals) with a 12-hour light/dark cycle.

\subsection{Pharmacological treatment}

At the end of week 8 of training, 14 SCT-trained rats and 4 SCTuntrained rats received daily i.p. administration of $5-\mathrm{HT}_{2 \mathrm{~A}-2 \mathrm{~B}-2 \mathrm{C}}$ receptor agonist ( \pm -1-2,5-Dimethoxy-4-iodophenyl-2-aminopropane hydrochloride, DOI, Sigma Aldrich, $0.12 \mathrm{mg} / \mathrm{kg}$, dissolved in saline), for a two-week period.

\subsection{Locomotor data analysis}

Locomotor recovery was evaluated by means of BBB rating score and Catwalk analysis (see Fig. 1B) performed 1 to $3 \mathrm{~h}$ after DOI treatment. The BBB rating scale (Basso et al., 1995) was performed by two independent observers blinded to training and treatment group, on the basis of videos taken weekly during spontaneous runs of the SCT rats in a corridor with a smooth horizontal floor and plexiglass wall $(120 \times 35 \times 20 \mathrm{~cm})$. The slope $(\mathrm{m})$ of the BBB curve was defined as follows: $\mathrm{m}=(\Delta \mathrm{y} / \Delta \mathrm{x}) \times 100$, expressed as a percentage, where $\mathrm{y}$ is the BBB score gain for $x$ number of days. If the BBB gain is 2 in one day, the slope for this period will be $200 \%$. The CatWalk system (CatWalk XT 9.1, Noldus Information Technology bv, Wageningen, The Netherlands) was used to evaluate gait parameters. All the characteristics of the system have been described previously (Cheng et al., 1997; Hamers et al., 2001; Khalki et al., 2013). The test was performed on 14 SCT- 
A

\section{Locomotor Animal groups Changes in MNs \\ assessment connectivity}
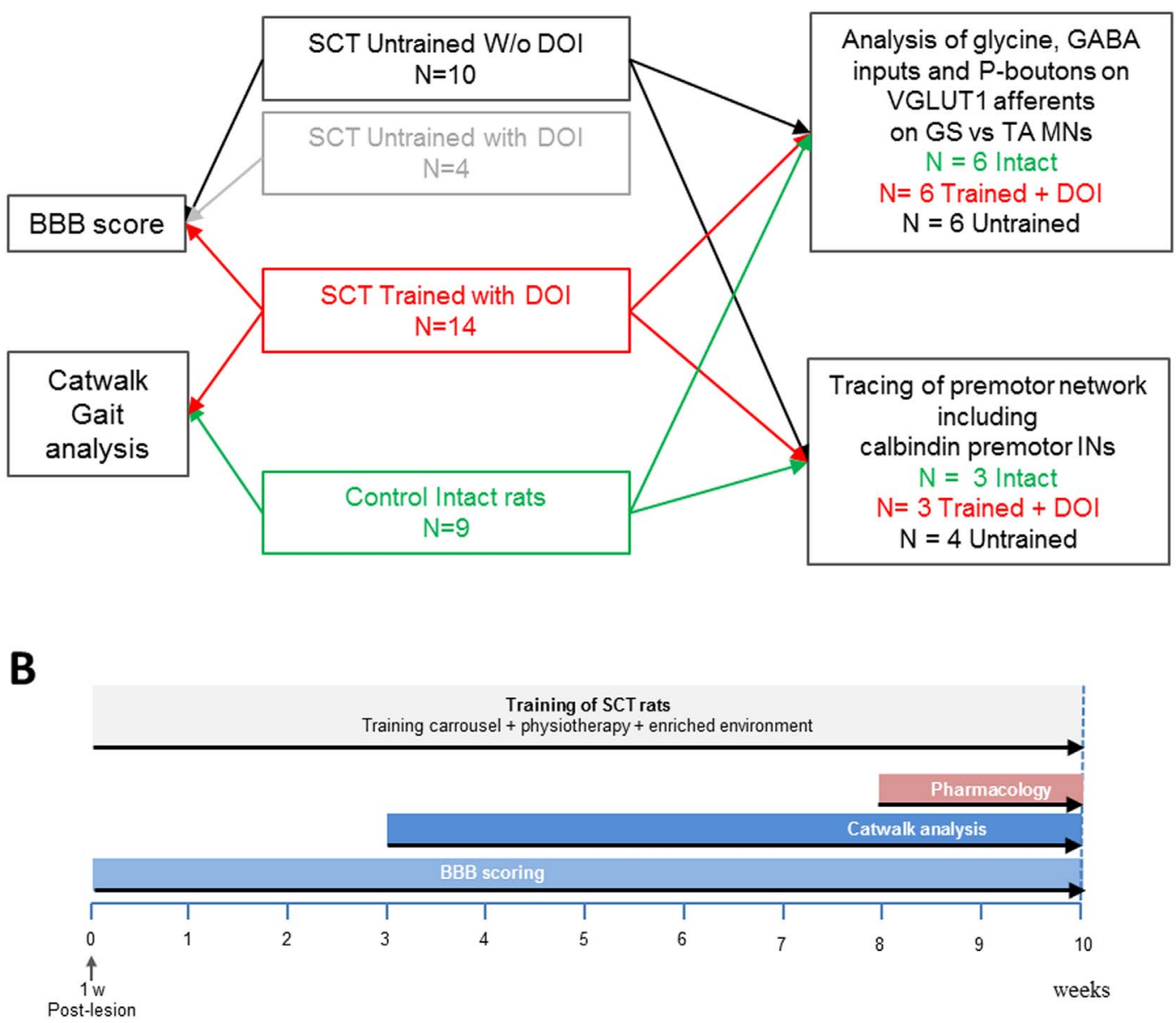

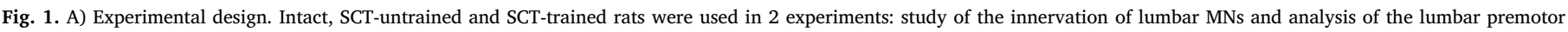
network using rabies virus. B) Time course of the exercise training, BBB scoring and Catwalk gait analysis and pharmacological treatment of SCT rats.

trained rats from the fourth week of training. Rats freely ran across a glass walkway located in a dark corridor. A combination of green LED lighting of the glass plate and red light illumination of the ceiling enabled visualization of the paw prints and the body contour of the animal which were recorded using a high-speed digital camera (100 frames/s) positioned beneath. At least three runs were collected for each session.

Catwalk analysis enabled quantitative assessment of quadruped locomotion, as soon as footprints of the hindlimbs were recordable (Hamers et al., 2001). The following parameters were analyzed:

Stride length: distance between successive placements of the same paw $(\mathrm{cm})$.

Stance phase: time of contact of the paw with the glass floor (sec).

Swing phase: time during which the paw is not in contact with the glass floor (sec).

Step cycle: time between two consecutive placements of a single paw (stance plus swing duration).

Phase dispersion: measures the synchrony of initial contact between pairs of limb. It is the timing between the initial contacts of paw pairs (i.e. RF-LH) expressed as a percentage of the step cycle of the anchor paw. For "in phase" limb pairing, the phase dispersion is $0 \%$. Limb pairs that alternate typically yield a phase dispersion of $50 \%$, out of phase.

Regularity index (RI): expresses the number of normal step sequence patterns (NSSP) relative to the total number of paw placement (PP), calculated as follows: $\mathrm{RI}=\mathrm{NSSP} \times 4 / \mathrm{PP} \times 100 \%$.

Stepping pattern: stepping patterns were classified as normal when the animal placed the four paws one after another in an alternate, cruciate or rotate pattern. Cruciate or rotate refer diagonal and lateral sequences (Gillis and Biewener, 2001), respectively, in the order of limb recruitment (Cheng et al., 1997).

\subsection{Retrograde labeling of motoneurons}

Adult rats were anaesthetized by i.p. administration of a mixture of ketamine (Imalgene, Merial, $60 \mathrm{mg} / \mathrm{k}$ ) and xylazine (Rompun, Bayer, $10 \mathrm{mg} / \mathrm{kg}$ ). They received bilateral injections of Fast blue (FB, $0.5 \%$ in $\mathrm{NaCl} 0.9 \%$, Sigma, $10 \mu \mathrm{L}$ ) in the TA muscle and Cholera Toxin $ß$ subunit-FITC $\left(1 / 100\right.$ in $\mathrm{H}_{2} \mathrm{O}$; CTb, Sigma Aldrich, $\left.20 \mu \mathrm{L}\right)$ in the lateral GS muscle. Optimal labelings were obtained after 7 days of retrograde transport of the dye, during which the training protocols were normally maintained.

\subsection{Transneuronal tracing of the premotor network with rabies virus $(R A B V)$}

The N2C variant of the challenge virus standard (CVS) strain of RABV was amplified in neuroblastoma cells (N2A) for 5 days at $33{ }^{\circ} \mathrm{C}$ (Fouquet et al., 2015) and the cell culture supernatant was concentrated as previously described (Coulon et al., 2011). Pilot experiments determined the optimal incubation time for the detection of RABV-infected MNs and premotor INs in the lumbar spinal cord (SC) of 
control rats after unilateral injection of the virus into GS muscle (3 sites of injection, $1.5 \times 10^{7} \mathrm{FFU}$ in $\left.3 \times 10 \mu \mathrm{L}\right)$. At $72 \mathrm{~h}$ p.i. $(n=2)$, RABVinfected MNs and a small number of premotor INs were detected along the L1 to L6 segments. Supplementary Fig. 1A shows a transverse section of the L4 segment where at most only a dozen infected INs can be observed. In L1 and L6 segments only a few infected INs were found. RABV-infected INs were mainly located ipsilateral to the injection (only $12.2 \pm 6 \%$ of contralateral RABV-infected INs). Our data is in agreement with early infection of INs mono-synaptically connected to MNs (Coulon et al., 2011; Ruigrok et al., 2008). At $80 \mathrm{~h}$ p.i. $(n=3)$, the number of RABV-infected INs increased significantly in the SC (Supplementary Fig. 1B). Most of the RABV-infected INs were located ipsilateral to the injection site, where their location very well matched the mapping of GS premotor INs reported by (Tripodi et al., 2011) when using monosynaptically restricted trans-synaptic virus. The brainstem remained devoid of infection, meaning that supraspinal neurons with long-descending tracts such as cells of the ventromedial reticular formation, which directly contact MNs (Holstege and Kuypers, 1987; Ruigrok et al., 2008), were not yet infected. At 88 h p.i. ( $n=4$, Supplementary Fig. 1C), an enormous increase in number of RABV-infected INs was observed and the tangle of strongly immunolabeled dendrites of both MNs and INs did not allow quantification in all the sections. Contralateral to the injection site, the infection largely extended to the dorsal horn. Therefore, some of the many infected INs observed at $88 \mathrm{~h}$ were undoubtedly di-synaptically connected to MNs. At this time, some neurons were observed in the ipsilateral lateral vestibular nucleus and in the ventromedial reticular formation, indicating that the infection had started to reach these supraspinal neurons with long-descending tracts connected mono-synaptically to MNs (Kasumacic et al., 2015; Ruigrok et al., 2008). In all, these data show that $72 \mathrm{~h}$ is an early stage of mono-synaptic infection of INs, and quantification at this time would result in a strong underestimation of the population of INs monosynaptically connected to MNs. In line with the estimated $12 \mathrm{~h}$ duration of the multiplication cycle of the virus (Coulon et al., 2011), $80 \mathrm{~h}$ can be considered as a late stage of mono-synaptic infection of INs although quantification at this time may include some INs that are di-synaptically connected to MNs. Yet, we are confident that a better estimation of the real population of INs connected to MNs can be determined at $80 \mathrm{~h}$ post-infection.

\subsection{Control of the absence of axon regeneration across the lesion in SCT- untrained and SCT-trained rats}

The completeness of the section was verified by anatomical magnetic resonance imagery (aMRI) processed $2 \mathrm{~h}$ after surgery (Supplementary Fig. 2A). To assess the absence of axonal regrowth across the lesion, we adapted the protocol previously described (Tillakaratne et al., 2010), with transneuronal retrograde transport of RABV (Coulon et al., 2011). We compared the extent of RABV infection in the spinal cord and brainstem of intact $(n=1)$, SCT-untrained $(n=2)$ and SCT-trained rats $(n=1)$, after inoculation of the virus in the GS muscle. After 5 days of infection, the intact rat displayed typical signs of rabies (ruffled fur, stoop, loss of weight, paralysis of hindlimbs). High densities of RABV-infected neurons were observed in the lumbar and thoracic spinal cord as well as in brainstem structures where vestibular and reticular nuclei were strongly labeled (Supplementary Fig. 2B). At 7 days post-infection (p.i.), which is lethal for intact rats, SCT-untrained and SCT-trained rats showed a paralysis of hindlimbs but an absence of rabies symptoms. Histological examination showed a high degree of infection below the lesion, but a complete absence of RABV-infected neurons in the thoracic spinal cord above the lesion, and in the brainstem (Supplementary Fig. 2C, D). These results clearly confirm the absence of axonal regeneration across the lesion in both SCT and SCT-trained rats, 11 weeks post-lesion.

\subsection{Perfusion and sectioning}

Animals were anaesthetized with sodium pentobarbital (Nembutal CEVA, $80 \mathrm{mg} / \mathrm{kg}$ ) 2 to $4 \mathrm{~h}$ after DOI treatment for pharmacologicallytreated rats and perfused intracardially with $100 \mathrm{~mL}$ of phosphate buffer saline (PBS $0.1 \mathrm{M}, \mathrm{pH}=7.4$ ), followed by $400 \mathrm{~mL}$ of $4 \%$ paraformaldehyde (in PB $0.1 \mathrm{M}, \mathrm{pH}=7.3$ ). To study the innervation of MNs, lumbar spinal cords were cryoprotected for $24 \mathrm{~h}$ in $30 \%$ sucrose and frozen at $-80^{\circ} \mathrm{C}$. The lumbar segments were serially cut in $30 \mu \mathrm{m}$ transverse sections using a cryostat (Microm) and collected in 48 well plates filled with cryoprotective solution and stored at $-20{ }^{\circ} \mathrm{C}$. To trace the premotor network, the whole lumbar spinal region was cut with a vibratome (Microm) in $30 \mu \mathrm{m}$ serial transverse sections collected in PBS with $\mathrm{NaN}_{2}(0,02 \%)$ in 48 well plates, and kept at $4{ }^{\circ} \mathrm{C}$. The 9 SCTtrained and pharmacologically treated animals used for these two series of experiments displayed final BBB scores $\geq 10$.

\subsection{Muscle mass assessment}

After perfusion, the left and right GS, TA and soleus (Sol) muscles were dissected, weighed, and then normalized to the total body weight of the animal in order to correct for inter individual variability (Fig. 3E).

\subsection{Immunohistochemistry}

We performed 3 series of experiments to study $i$ ) the expression of Pboutons on VGLUT1 sensory afferents in contact with GS and TA MNs, ii) the expression of GABA and glycine axon terminals apposed on GS and TA MNs and iii) the network of lumbar INs premotor to GS MNs. We prepared the following cocktails of primary antibodies (Ab): (i) guineapig anti-Vesicular Glutamate Transporter 1 (VGLUT1, AB5905, Millipore, 1:5000; (Brumovsky et al., 2007; Todd et al., 2003), mouse anti-65KDa isoform of glutamate decarboxylase (GAD65, MAB351R, Millipore, 1:100) and rabbit anti-cation-chloride co-transporter (KCC2, AB07432, Millipore, 1:400 (Boulenguez et al., 2010) Abs. (ii) guineapig anti-glycine transporter (GlyT2, AB1773, Millipore, 1:20,000) and mouse anti-GAD65 (MAB351R, Millipore, 1:100) Ab. Used at a high concentration, GAD65 Ab enabled to detect both P-boutons apposed on VGLUT1 axon terminals which were intensely immunoreactive (Kapitza et al., 2012), but also GABAergic axon terminals directly contacting MNs (Esclapez et al., 1994; Lorenzo et al., 2014). (iii) mouse anti-RABV phosphoprotein Ab (1:10,000; Coulon et al., 2011) associated with goat anti-choline acetyl transferase (ChAT, AB144P, Millipore, 1:100) Ab for identification of MNs or associated with rabbit anti-calbindin Ab (CB38, Swant, 1:2000) to characterize sub-populations of premotor Renshaw cells (Alvarez et al., 2005; Carr et al., 1998; Coulon et al., 2011). These Abs were revealed with species-specific donkey secondary Abs conjugated to Alexa Fluor 488 (1:400; Invitrogen), Cy3 or Cy5 (1:400; Jackson ImmunoResearch). For each experiment, sections from intact, SCT-untrained (11 week post-lesion) and SCT-trained rats were incubated with the same solution of primary antibodies, and each experiment was repeated at least three times with three different groups of rats ( 3 rats per group). As negative controls, in each series of double or triple immunolabelings, we omitted each primary antibody and observed a complete absence of labeling with the appropriate secondary antibody.

\subsection{Analysis of the distribution of RABV-infected premotor INs in the lumbar spinal cord}

The distribution of RABV-infected INs premotor to GS MNs and calbindin-labeled INs in the lumbar spinal cord (L1 to L6) of intact, SCT-untrained and SCT-trained rats was reconstructed from serial transverse sections (1 out of 12) with Neurolucida software (Microbrightfield Inc., Williston, VT) using a fluorescence microscope 
(Nikon 90i). The location of the RABV-labeled premotor INs, calbindin ${ }^{+}$neurons and $\mathrm{RABV}^{+} \mathrm{MNs}\left(\mathrm{ChAT}^{+}\right)$were plotted on charts of transverse sections of the lumbar segments adapted from (Molander et al., 1984). The Renshaw cells were identified by their immunoreactivity to calbindin and RABV and their location in the ventral horn; i.e. within lamina IX or the part of lamina VII directly adjacent to IX and always within $100 \mu \mathrm{m}$ from the inner edge of the ventral funiculus (Alvarez and Fyffe, 2007; Alvarez et al., 2005). We used a Kruskal-Wallis ANOVA test to compare the number of RABV-labeled premotor INs through the lumbar segments, the ratio premotor INs/ MNs, the proportion of premotor INs ipsi and contralateral to the injection site and the number of Renshaw cells, in the three groups of rats. To estimate the spatial distribution of the premotor INs, we computed and displayed the color-coded two-dimensional histogram of the neuron positions in each segment of the spinal cord. Using a regular square grid on the plane, we determined the mean number of neurons into each square that was coded using a color scale (Fig. 6F) or pointed (Fig. 7F). Squares containing no labeled neurons were left blank.

\subsection{Quantification of innervation of lumbar motoneurons}

We used laser scanning confocal microscopy (LSM ZEISS 510 Meta) to quantify the glutamatergic (VGLUT1) inputs and their associated Pboutons, as well as glycinergic and GABAergic inputs onto retrogradely identified GS and TA MNs of intact, SCT-untrained and SCT-trained rats ( $n=3$ rats per group). Only MNs showing a clear nucleus were scanned in stacks of $0.2 \mu \mathrm{m}$-thick optical sections using a $63 \times$ oil objective. Morphometric analysis was performed using Zen Software (version 2012). GS MNs in the 3 groups of rats had similar perimeters (Fig. 5C, $P=0.48$, ANOVA Kruskal Wallis test). We calculated the density of each type of axon terminal (number of axon terminal per $100 \mu \mathrm{m}$ of motoneuronal plasma membrane), and measured the area of GABAergic, glycinergic and VGLUT1 axon terminals contacting the MNs and the area of presynaptic P-boutons as well as their density (mean number of P-boutons per VGLUT1 terminal). P-boutons were spheroid elements intensely immunolabeled with an area significantly larger than the clusters of CTb-FITC identifying the GS MNs $(1.2 \pm 0.03 \mu \mathrm{m}$ and $0.9 \pm 0.03 \mu \mathrm{m}$, respectively, $P<0.0001)$. The morphometric parameters were compared using Mann Whitney test. For quantification of the levels of expression of KCC2 in the membrane of lumbar MNs, we digitized intensity measurements using Zen Software as previously described (Bos et al., 2013). Measurements were compared in the three groups of rats using a Kruskal Wallis (ANOVA) test followed by Dunn's post hoc test. $P<0.05$ was considered significant.

\subsection{Principal component analysis}

To decrease the high-dimensionality of our data, i.e. 8 variables describing the morphometry of the innervation of GS versus TA MNs, we performed principal components (PC) analysis using the Factomine $\mathrm{R}$ and Psych packages (The R Foundation for Statistical Computing, Institute for Statistics and Mathematics, Wien, Austria). PC analysis is defined as an orthogonal linear transformation of the dataset into a reduced subspace with new coordinates, such that the variance is maximized on each new coordinate axis (Field et al., 2012). By reducing a data set from a group of interrelated variables into a smaller set of factors, PC analysis achieves parsimony by explaining the maximum amount of common variance in a correlation matrix with the smallest number of factors. Initial analysis was run to obtain eigenvalues for each component in the data and did not reveal fundamental differences between initial extractions or orthogonal rotation of PC. We usually kept the first two PCs with Kaiser's criterion over 1 and their combination explained about 40 to $50 \%$ of the variance (Field et al., 2012). We kept the factor loadings over $|0.7|$ in PC analyses which were compared by using non-parametric analyses (Mann-Whitney $U$ tests, $P<0.05)$.

\section{Results}

\subsection{Stepping ability of SCT-trained rats}

The exercise training started one week after transection and was performed every day (Fig. 1B). The procedure combined daily sessions of free moving in an enriched environment, sessions of imposed locomotion in an automatic training carrousel and physiotherapy exercises alternating flexion and extension of both hindlimbs (Video 1). Locomotor recovery was evaluated using the behavioral BBB rating scale (Fig. 2) and Catwalk gait analysis (Fig. 3).

$B B B$ scoring: Immediately after SCT, all rats displayed a complete loss of hindlimb function (Fig. 2, Video 2). After 4 weeks of training, SCT-trained rats displayed extensive movements of one or two joints of the hindlimbs (BBB score $=2$, Fig. 2). During weeks 5 to 8 , the BBB score increased with a constant slope $(\mathrm{m}=100 \%)$ to reach a mean value of $6.1 \pm 0.4$, reflecting movements of all three joints of the hindlimbs, with extensive movements of at least two joints (Fig. 2, see Video 2 at 54 days training).The i.p. treatment with the $5-\mathrm{HT}_{2} \mathrm{R}$ agonist DOI started at the beginning of week 9 and was performed daily during the two following weeks, in combination with the exercise training. This resulted in a marked increase of the slope of the BBB curve $(\mathrm{m}=310 \%)$. After one week of DOI treatment, the BBB score reached $9.1 \pm 0.6$ (Fig. 2) meaning that the animals displayed frequent weight bearing of the hindlimbs, although the stance phase was occasionally performed on the dorsal part of the paw. After an additional week of DOI treatment, the rats showed frequent plantar placement of the hindpaws and consistent body weight bearing (BBB score $10.3 \pm 0.3$, Video 2), which was also visible during stairs climbing (although not taken into account in BBB scoring).

To unravel the respective role of training alone and DOI treatment on the improvement of motor capacities, a group of 3 rats received exercise training without pharmacological treatment during 9 weeks (Supplementary Fig. 3). During week 9 of training, the slope of their BBB curve was notably less steep (50\%) than that of trained animals between week 4 and week $8(\mathrm{~m}=100 \%)$ BBB score reached $6.6 \pm 0.9$ (note that this slope was much weaker than the one of animals pharmacologically treated during this period (300\%, red curve). Then, these trained animals received daily injections of DOI during 2 weeks, in combination with exercise training (orange curve). After 1 week of treatment, the slope of the BBB curve increased by $265 \%$, in parallel with the slope of the group pharmacologically treated during week 9 $(P=0.0002$, BBB score $9.2 \pm 0.5)$. After an additional week of treatment, the BBB score reached $10.5 \pm 0.3$.

As previously reported, the absence of re-establishment of supraspinal connections resulted in an absence of forelimb-hindlimb coordination, which limits the upper score reachable in the BBB scale to 11 (Antri et al., 2002). For control experiments, a group of SCT-untrained rats received a DOI treatment during weeks 10 and 11 after the lesion. These animals displayed a weak BBB score (1.3 \pm 0.4$)$, significantly smaller than observed after training combined with DOI treatment $(P=0.0019)$ but not different from SCT-untrained and nontreated animals $(P=0.43$; Fig. 2$)$.

\subsection{Catwalk gait analysis}

As previously stated (Hamers et al., 2001), Catwalk data can be acquired only from the time that the animals regained at least frequent weight supported stepping (BBB 9 or higher). Indeed, during the first 7 weeks of training, no hindlimb footprints could be recorded from the Catwalk system, as well as for SCT-untrained rats at all times (Fig. 3A1, B1, B2). During week 8 of training, before DOI treatment, separate footprints of the hindlimbs could be recorded, but they were irregular (Fig. 3B3). After one week of DOI treatment, regular footprints were recorded but they were characterized by a dorsal foot placement (Fig. 3A2, B4). Then, at the end of the second week of treatment, the 


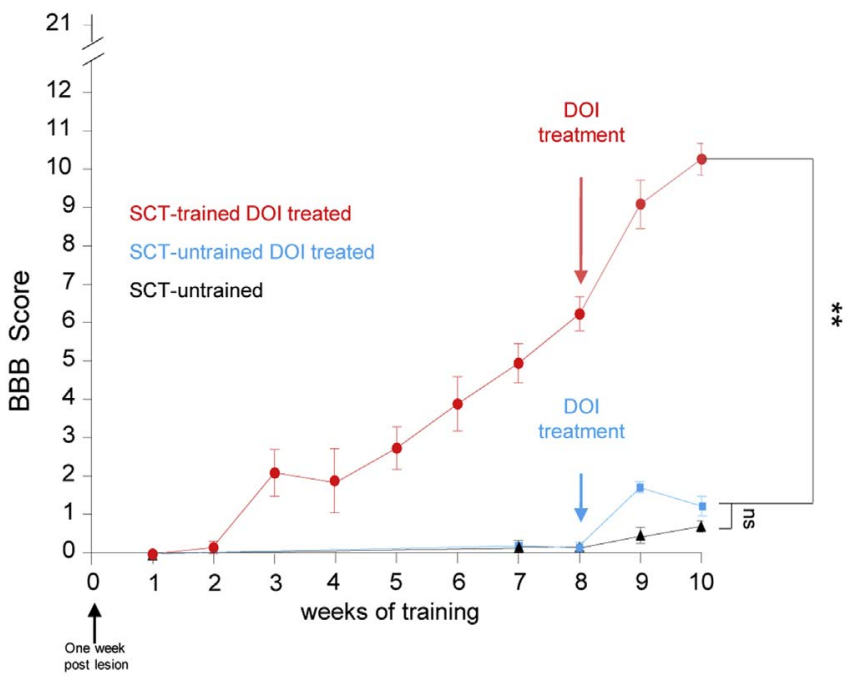

Fig. 2. Motor performances of trained-rats treated with DOI (red line), SCT-untrained rats treated with DOI (blue line), and SCT-untrained and non-treated rats (black line), assessed by BBB scoring. ${ }^{*} P<0.05 ;{ }^{* *} P<0.01$; ${ }^{* * *} P<0.001$, ns: non-significant.

rats, hereafter referred to as SCT-trained rats, performed runs that very much resembled those of intact rats (Fig. 3B6). Although SCT-trained rats displayed normal step sequence patterns, these were not always executed in a smooth, uninterrupted way as shown by the regularity index, which decreases from $100 \%$ in fully coordinated intact rats to 92\% (Fig. 3C1, $P=0.12$ ), thus indicating an incomplete interlimb coordination. This incomplete coordination was mainly due to a significant impairment of the forelimb-hindlimb coordination as shown by the measurement of the phase dispersion (PD): in both intact and SCTtrained rats, PD value was close to $50 \%$ for both fore and hindlimb girdle pairings, indicating a typically alternate stepping (Fig. 3C2, $P=0.77$ ). On the other hand, in SCT-trained rats, PD was strongly impaired for diagonal and ipsilateral pairings as compared to intact ( $P \leq 0.0001$ and $P=0.0028$, respectively, Fig. 3C2). In normal rats, the frequency of regular patterns included alternate $(85.2 \%$ of the patterns) and cruciate (14\%) stepping patterns, but only rarely rotate pattern (0.8\%, Fig. 3C3, see also Fig. 3D). In SCT-trained rats, the frequency of regular patterns was modified: they used an alternate pattern in $65 \%$ of the runs, a cruciate pattern in $15 \%$, and a rotate stepping pattern in $19 \%$ of the runs (Fig. 3C3). Examination of the static and dynamic gait parameters revealed that SCT-trained rats displayed fore and hind strides length similar to those of intact rats (Fig. 3C4, $P=0.07$ and $P=0.33$, respectively). The forelimb step cycle was similar to control (Fig. 3C5), although it resulted from an increase of the stance phase (126\% of the controls, Fig. 3C6) and a decrease of the swing phase (80\% of the controls; Fig. 3C7). The hindlimbs step cycle was significantly increased compared to controls $(P \leq 0.0001$, Fig. 3C5), and resulted from a reduction of the stand phase ( $80 \%$ of the controls, $P=0.003$, Fig. 3C6) and an increase of the swing phase duration (213\% of the controls, $P \leq 0.0001$, Fig 3C7). It is interesting to note that the weight of hindlimb muscles significantly dropped down in SCT rats, and returned to normal values in SCT-trained rats (Fig. 3E).

\subsection{Morphometric changes of the innervation of lumbar MNs in SCT- untrained and SCT-trained rats}

The morphometric analysis of density and area of excitatory and inhibitory axon terminals in contact with retrogradely identified GS and TA MNs ( $N=106$ and 129, respectively) is summarized in Table 1 for the 3 groups of rats (intact, SCT-untrained and SCT-trained rats). In a first set of experiments, we analyzed the excitatory primary sensory afferents (VGLUT1-immunoreactive axon terminals, $N=600$ ) apposed on the cell bodies of MNs and we quantified the axo-axonic presynaptic
P-boutons (GAD65-immunopositive, $N=1320$ ) apposed on these VGLUT1 inputs (Fig. 4A). Then, we quantified the inhibitory glycinergic $(N=2334)$ and GABAergic terminals $(N=669)$ directly apposed onto GS and TA MNs somata (Fig. 4A, B).

\subsubsection{Intact versus SCT-untrained rats}

In intact rats, VGLUT1 terminals were large boutons (Figs. 4A, 5A1) sparsely distributed on the cell bodies of GS and TA MNs with a mean density of $3.8 \pm 0.3$ and $3.7 \pm 0.4$ bouton per $100 \mu \mathrm{m}$ of plasma membrane of GS and TA MNs, respectively (Fig. 5A2), in line with the densities reported in sacral MNs (Kitzman, 2007). Each VGLUT1 axon terminal was contacted by an average of 1.7 P-boutons for GS MNs and 2.1 P-boutons for TA MNs (Fig. 5A3), in line with the densities of Pboutons/VGLUT1 terminal reported by two earlier studies (Hughes et al., 2005; Kapitza et al., 2012). In both GS and TA MNs, 5\% of the primary afferents did not receive any P-bouton (Fig. 5B1, B2). In SCTuntrained rats, the area of the VGLUT1 afferents was similar to controls in both GS and TA MNs (Fig. 5A1). Their density decreased in TA MNs $(P=0.008$; Fig. 5A2), but not in GS $(P=0.32)$. The mean density of $\mathrm{P}-$ boutons per VGLUT1 terminal and their area were unchanged (Fig. 5A3), although the percentage of VGLUT1 terminals free of any Pbouton increased to $18 \%$ on GS and $12 \%$ on TA MNs (Fig. 5B1, B2).

In intact animals, the glycinergic axon terminals represented $80 \%$ of the inhibitory inputs (Glycine + GABA) on GS MNs and $75 \%$ on TA MNs. In SCT-untrained rats, the proportion of glycinergic inputs decreased to $67 \%$ on both types of MNs, due to an increase of GABA inputs which was significant in GS MNs but not in TA MNs (Fig. 5A7), while the densities of Glycine inputs remained unchanged (Fig. 5A5). The area of GABAergic terminals was unchanged in both GS and TA MNs (Fig. 5A8), while the area of glycinergic terminals was stable on GS, but significantly decreased in TA MNs (Fig. 5A6).

\subsubsection{Intact versus SCT-trained}

In SCT-trained rats, the area of VGLUT1 terminals was significantly larger than in controls, on both GS and TA MNs $(P=0.002$ and $P=0.02$, respectively; Fig. 5A1), while their density did not significantly increase (Fig. 5A2). On both types of MNs, the area of Pboutons significantly increased compared to controls $(P=0.019$ and $P=0.04$, respectively; Fig. 5A4) as well as the density of P-boutons per VGLUT1 terminal $(P=0.006$ and $P=0.01$, for GS and TA MNs respectively, Fig. 5A3). Interestingly, all the VGLUT1 primary afferent terminals were contacted by at least one P-bouton on both GS and TA MNs (Fig. 5B1, B2).

Glycine and GABA inputs displayed major morphometric changes in SCT-trained rats, compared to intact animals: the density of glycinergic and GABAergic axon terminals significantly increased on GS $(P=0.003$ and $P=0.02)$ and TA MNs $(P=0.03$ and $P<0.001$, Fig. 5A5, A7), while their mean area significantly decreased $(P \leq 0.0001$ and $P=0.0003$ on GS MNs; $P \leq 0.0001$ and $P \leq 0.0001$ on TA MNs, Fig. 5A6, A8).

\subsubsection{SCT-untrained rats versus SCT-trained}

We observed a major impact of training and pharmacology on the innervation of lumbar MNs (Table 1). Compared to SCT animals, SCTtrained rats' MNs received significantly higher densities of VGLUT1 primary afferent inputs $(P=0.007$ in GS and $P=0.006$ in TA), which were contacted by a higher density of P-boutons $(P<0.0001$ in GS and $P=0.009$ in TA). SCT-trained animals displayed significantly higher densities of glycinergic inputs on both GS $(P<0.0001)$ and TA MNs $(P=0.018)$, whereas the densities of GABA inputs were unchanged. On both types of MNs, the area of glycinergic and GABAergic terminals was significantly smaller than in SCT rats $(P<0.0001$ in all cases, Fig. 5).

\subsubsection{Expression of KCC2 at the membrane of lumbar MNs}

Because the hyperpolarizing effect of GABA and glycine depends on 

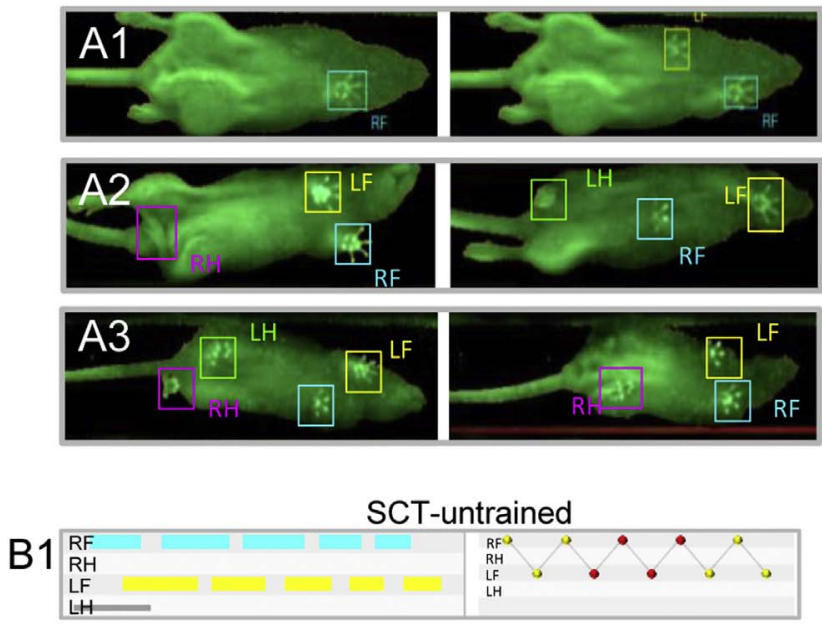

SCT-trained week 3

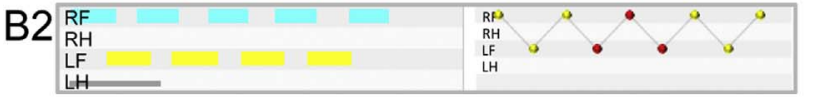

SCT-trained week 8

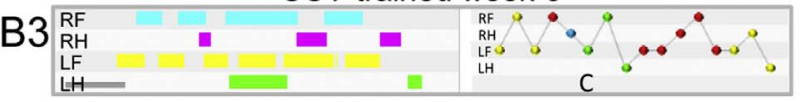

SCT-trained + DOI week 9

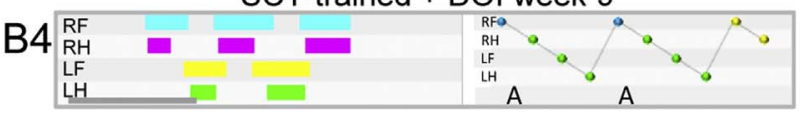

SCT-trained + DOI week 10
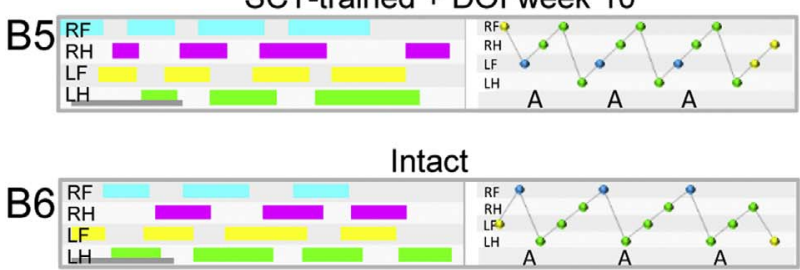

C1

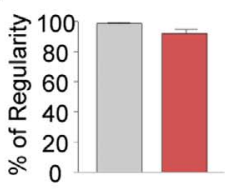

C3

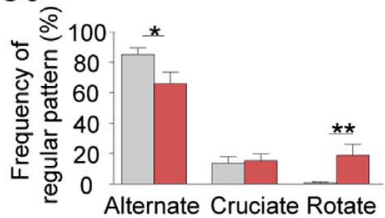

C5

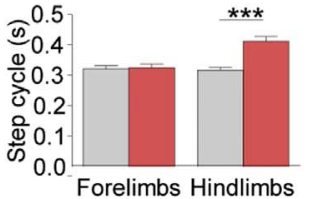

C7

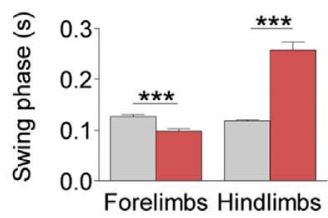

E

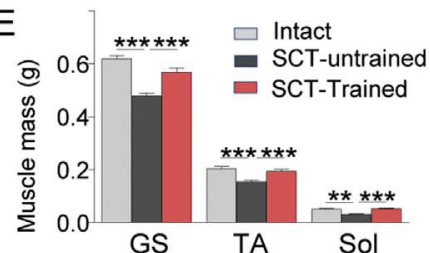

$\mathrm{C} 2$

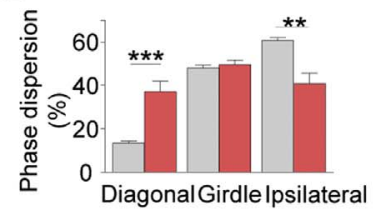

C4

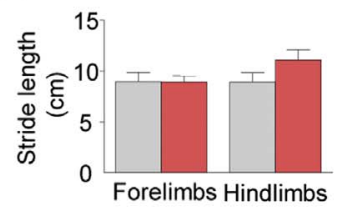

C6

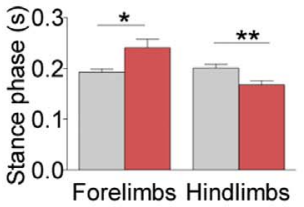

Intact $\square$ SCT-Trained

D
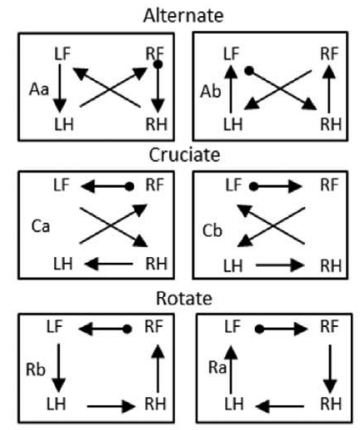

(adapted from Cheng et al., 1997)

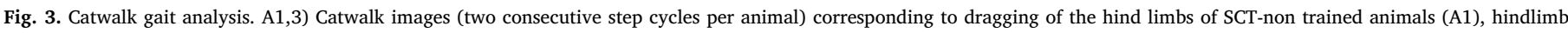

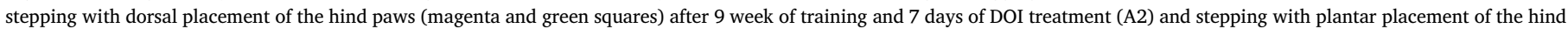

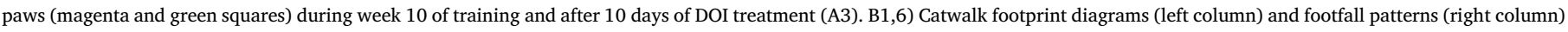

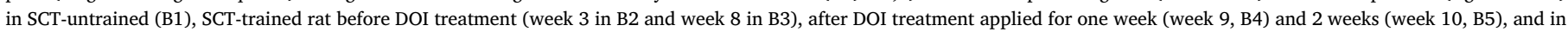

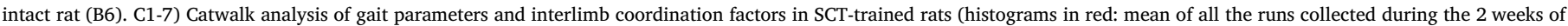

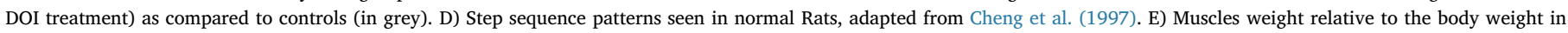

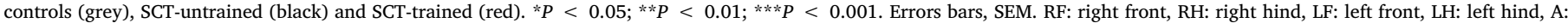
alternate pattern, C: cruciate pattern. GS: gastrocnemius, TA: tibialis anterior, Sol: soleus.

the level of expression of cation-chloride co-transporter KCC2 at the neuronal membrane (Doyon et al., 2016), we examined the expression of KCC2 in lumbar MNs of the three groups of rats. We observed a significant reduction of KCC2 expression in SCT-untrained rats compared to controls $(P<0.0001$, Fig. 5D). Conversely, in the lumbar MNs of SCT-trained rats, the levels of expression of KCC2 returned to intact values (Fig. 5D).

3.3.5. Principal component (PC) analysis of the innervation of lumbar MNs

Taking in account all the variables of the innervation of lumbar MNs in the three groups of animals (Fig. 5E) PC analysis indicated a clear separation between SCT-trained rats and the two other groups along the PC1 axis (accounting for $32.6 \%$ of the variance; $U=0$; $P<0.05$, respectively), while intact and SCT-untrained rats were not differentiated $(\mathrm{U}=4 ; P=0.21$ ). The extraction of the highest factor loadings (IvalueI $>0.7, P<0.05$ ) revealed that the parameters of presynaptic and postsynaptic inhibitory innervation of GS MNs contributed the most to separate SCT-trained rats from the others along the PC1 axis (Fig. 5F).

\subsection{Distribution of premotor INs in SCT-untrained and SCT-trained rats}

Upstream of the innervation of lumbar MNs, we compared the network of premotor INs in the three groups of rats by means of retrograde transneuronal tracing with RABV (Fig. 6A). Since parameters of innervation of extensor GS MNs were first involved in the differences between SCT-trained rats and the two other groups, we analyzed the distribution of premotor INs connected to GS MNs in intact $(n=3)$, SCT-untrained $(n=4)$ and SCT-trained rats $(n=3)$. The GS MNs characterized by their double immunolabeling $\mathrm{RABV}^{+}$and $\mathrm{ChAT}^{+}$ (Fig. 6B) were distributed within the L4 and rostral part of L5 segments (Fig. 6C). The number of infected MNs was not significantly different in the three groups of rats (data not shown). Premotor INs $\left(\mathrm{RABV}^{+}\right.$only) were distributed along all lumbar segments (L1-L6, Fig. 6C). The ratio premotor INs/MNs was also very close in the two groups $(593 \pm 157$ and $578 \pm 167$, in SCT-trained and intact rats, respectively, Fig. 6D). In SCT-untrained rats the ratio premotor INs/MNs $(235 \pm 102)$ decreased compared to both intact and SCT-trained rats, although nonsignificantly. 
Table 1

Morphometry of the innervation of GS and TA MNs: Statistical analysis of the area and density of VGLUT1 inputs, P-boutons, GABA and glycine axon terminals apposed on GS and TA MNs in intact, SCT-untrained (SCT) and SCT-trained rats (SCT-recovered).

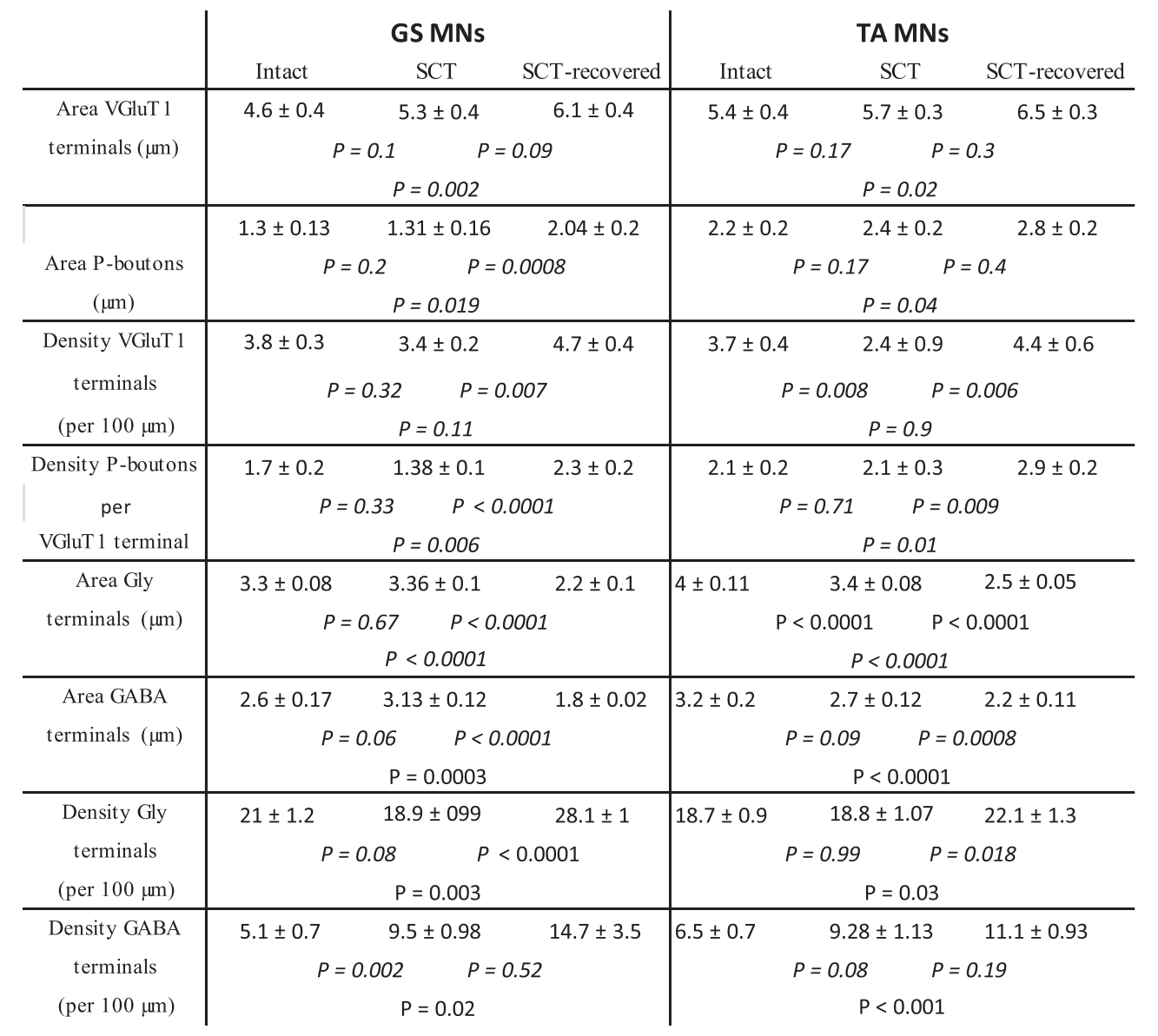

We then compared the distribution of premotor INs located ipsi and contralateral to the virus injection site. In intact rats, $26.2 \% \pm 1.43$ of the premotor INs were located contralaterally and no significant change was observed after SCT $(23.5 \% \pm 2.3, P=0.35$, Fig. 6 E). In SCTtrained rats, the population of contralateral premotor INs was significantly increased compared to both intact and SCT-untrained rats (30.3\% $\pm 1.7 ; P=0.04$ and $P=0.02$, respectively, Fig. $6 \mathrm{E})$. To analyze the rostro-caudal distribution of premotor INs, we computed heat maps of premotor INs density within each Rexed lamina of each lumbar segment (Fig. 6F). In intact rats, hot spots of premotor INs were observed ipsilateral in the medial part of laminae V-VII, and contralateral in the medial part of lamina VII and in the dorsal part of lamina VIII, along L1-L5 segments (Fig. 6F, left column). In SCT-trained rats the distribution of hot spots of premotor INs was similar to intact animals, although a hot spot of premotor INs was observed in the ipsilateral superficial laminae of L4 segment (Fig. 6F, right column). In SCT-untrained rats, the surface of the hot spots was reduced compared to the other groups (Fig. 6F, middle column). Non-parametric analyses showed that differences in local distribution of premotor INs between groups were observed only within L4 and L5 segments. Comparisons of intact and SCT-trained (Fig. 6F, right column) groups attested to a significant increase of the percentage of premotor INs in the ipsilateral superficial lamina I-III as well as in the contralateral lamina V of SCTtrained rats compared to intact animals. They also showed a decrease of premotor INs densities in the ipsilateral lamina VII in L4 segment of these animals. These analyses also emphasized numerous local deficit of ipsi and contralateral premotor INs in SCT-untrained rats compared to SCT-trained animals in L4 and L5 segments.
To unravel the issue of the significant increase of the density of inhibitory inputs observed on lumbar MNs of SCT-trained rats we studied the distribution of inhibitory premotor INs expressing calbindin (Druga, 2009) which include the sub-population of Renshaw cells also characterized by their ventro-medial location in the ventral horn (Alvarez et al., 2005), Fig. 7A). The mean number of calbindin ${ }^{+}$premotor INs was not significantly different in the 3 groups of rats (Fig. 7B, C) as well as the mean number of Renshaw cells synaptically connected to GS MNs (Fig. 7D). We then examined the proportion of calbindin ${ }^{+}$ premotor INs projecting ipsi or contralateral to the injection site. In SCT-untrained rats, the percentage of contralateral calbindin ${ }^{+}$premotor INs decreased, although not significantly, compared to intact rats $(18.8 \% \pm 4.1$ and $30.8 \% \pm 6.1$, in SCT-untrained $v$ s intact, respectively, $P=0.08$, Fig. 7E). In SCT-trained rats, the population of contralateral calbindin ${ }^{+}$premotor INs $(32.4 \% \pm 3.7)$ was similar to intact rats $(P=0.28)$ and significantly increased compared to SCT-untrained rats $(P=0.03)$. We computed a mapping of calbindin ${ }^{+}$premotor INs in the different laminae of the 6 lumbar segments for the three groups of rats (Fig. 7F). Non-parametric analyses showed that, in L4 and L5 segments, SCT-trained animals displayed an increase of calbindin ${ }^{+}$ premotor INs in the contralateral laminae VII and VIII but a decrease in the ipsilateral lamina VIII compared to SCT-untrained rats.

In sum, these results show that 1 ) The ratio INs/MNs decreased in SCT-untrained rats, but returned to normal values in SCT-trained rats. On the other hand, the percentage of contralateral premotor INs was significantly higher in SCT-trained rats than in the two other groups. 2) In SCT-untrained rats, the percentage of commissural calbindin ${ }^{+}$premotor INs was reduced compared to controls. In SCT-trained rats this 

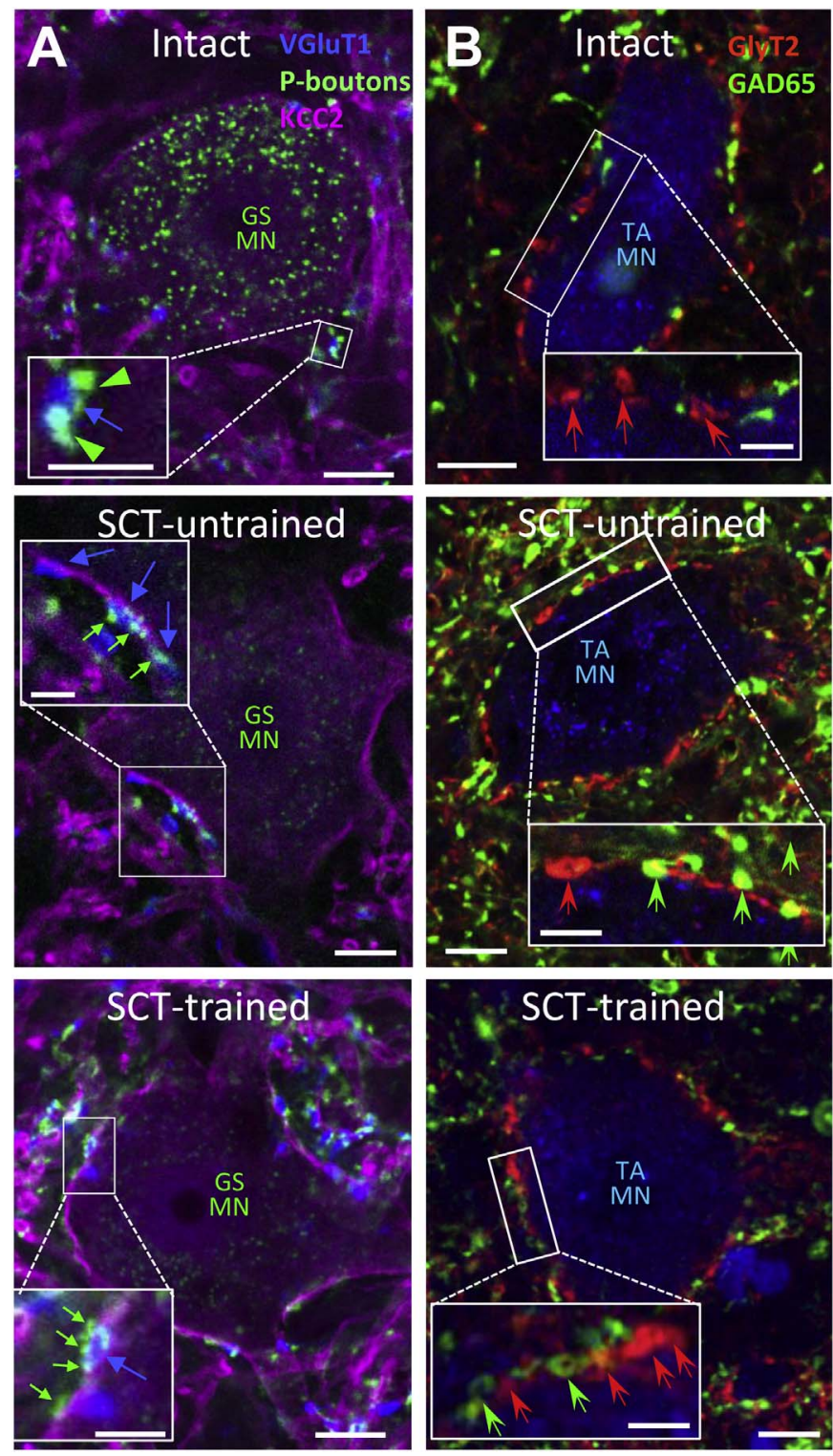

Fig. 4. A) Single confocal optical section of triple immunolabeling of primary Ia afferent terminals (VGLUT1, blue) and their presynaptic P-boutons (GAD65, green) on the plasma membrane (KCC2, purple) of GS MNs retrogradely identified with CTb-FITC, in intact, SCT-untrained and SCT-trained rats. B) Immunolabeling of glycinergic (GlyT2, red) and GABAergic (GAD65, green) axon terminals on TA MNs retrogradely identified with fast blue in the three groups of rats. Scale bars: $10 \mu \mathrm{m}$; insets: $5 \mu \mathrm{m}$.

percentage was close to normal values and the significant differences between SCT-untrained and SCT-trained rats were mainly due to a gain of commissural calbindin + premotor INs in contralateral laminae VII and VIII and a reduction of calbindin + premotor INs in ipsilateral lamina VIII.

\section{Discussion}

This work combines for the first time the study of the plasticity of innervation of lumbar MNs and the organization of premotor network connected to these MNs after training of SCT rats. The main results are summarized in a graphical abstract.

The training protocol designed for this study was based on existing data showing that neurorehabilitative strategies that combine several approaches are key to an efficient locomotor recovery (Courtine et al., 2009; Musienko et al., 2011). The protocol adapted here, combined early and long-lasting multi exercise training with delayed pharmacological treatment with a 5HT agonist. This resulted in the restoration of unassisted overground stepping, which has been previously shown in rats transected at the neonatal stage (Miya et al., 1997), but not in animals transected at adulthood (Weber and Stelzner, 1977).

Therefore, the changes of neural connectivity below the lesion have been studied as the result of a multi system rehabilitation protocol and the question of analyzing the respective role of exercise training and pharmacological treatment has not been broached. On the other hand, controlling the most effective timing of administration of DOI during the exercise training turns out to be of particular importance. On the one hand, it has been shown that chronic treatment of SCT rats with 5$\mathrm{HT}_{2} \mathrm{Rs}$ agonists starting shortly after the lesion has only limited effect on body weight bearing (Antri et al., 2002). On the other hand, the present results showed that in absence of training, two weeks of daily DOI treatment starting 9 weeks after lesion has no beneficial effects on locomotion recovery. Furthermore, we show that training alone, implemented for a period of 8 (Fig. 2), or 9 weeks (Supplementary Fig. 3) results in a constant improvement of the rat's locomotor capacities, but DOI treatment performed at the end of both trainings significantly strengthens the BBB score of the animals. Therefore, we suggest that exercise training and $5-\mathrm{HT}_{2} \mathrm{R}$ agonists treatment synergically interact to enhance recovery of unassisted overground stepping in transected rats, but training is a prerequisite for pharmacological treatment to be effective.

\subsection{Disorganization of the premotor networks and reduction of inhibition on MNs in SCT-untrained animals}

We show that in SCT-untrained rats there is a decrease to one third of the number of INs connected to the RABV-infected lumbar GS MNs (Fig. 6D). Since the input density on lumbar GS MNs was not reduced in SCT-untrained animals, the reduction of the last-order population suggest either an axonal sprouting of the remaining premotor INs on the cell bodies of lumbar MNs, or a specific loss of premotor INs impinging on dendrites. This second suggestion is consistent with the decrease of dendritic branches reported on MNs of lesioned rats (Kapitza et al., 2012; Wang et al., 2015). Although the relationship between the density of synaptic inputs and their efficiency cannot be established on the basis of anatomical data alone, our data strongly suggest a decrease in strength of inhibition on lumbar MNs in SCT-untrained rats. First, whereas in intact rats, $5 \%$ of the VGLUT1 afferents were deprived of GABAergic P-boutons, in line with previous data (Hughes et al., 2005), in SCT-untrained rats the percentage of VGLUT1 axon terminals free of any P-bouton was substantially increased (12 to 18\%), as also reported in rats with sacral transection (Kapitza et al., 2012). Second, in SCTuntrained rats, we observed a significant increase of the density of GABAergic inputs onto lumbar MNs, while the density of glycinergic inputs remained stable. These changes in the proportion of GABA/ glycine inputs are in agreement with the reorganization of the expression of GABA and glycine receptors reported in lumbar MNs of transected rats (Khristy et al., 2009; Sadlaoud et al., 2010). However, as previously reported (Boulenguez et al., 2010), we show a significant down-regulation of KCC2 at the membrane of lumbar MNs of SCT-untrained rats, thereby depolarizing the $\mathrm{Cl}^{-}$equilibrium potential and reducing the strength of postsynaptic inhibition. Consequently, in line with the post-lesional up-regulation of GAD 67 levels (Tillakaratne et al., 2002), the higher density of inhibitory inputs onto lumbar MNs observed after SCT must result in a mechanism of disinhibition (Boulenguez et al., 2010). Among the various mechanisms responsible for the hyperexcitability of spinal reflexes after SCT, these data underlie the roles of a reduction of presynaptic inhibition and a decrease of direct inhibition onto lumbar MNs, which might be associated with malfunctions such as chronic pains or spasticity (Boulenguez et al., 2010; Kapitza et al., 2012). 
A1

A2

A3

A4
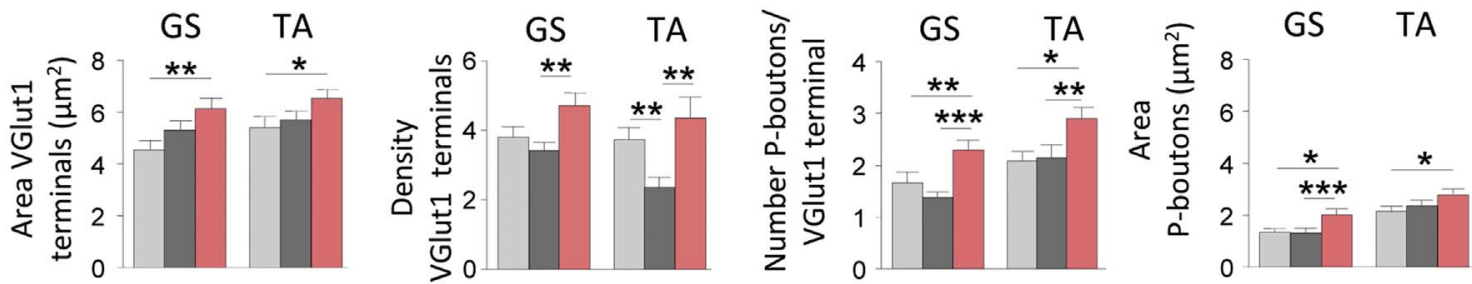

A5

A6

A7

A8
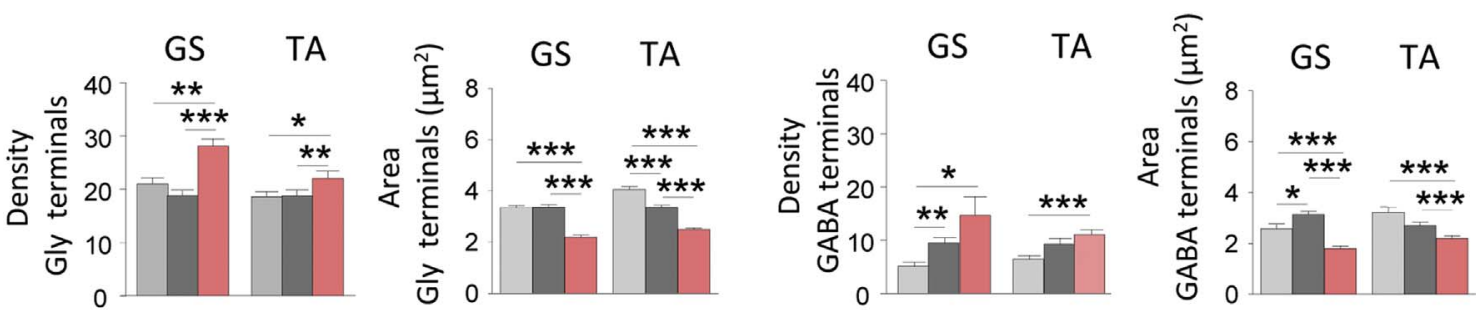

B1

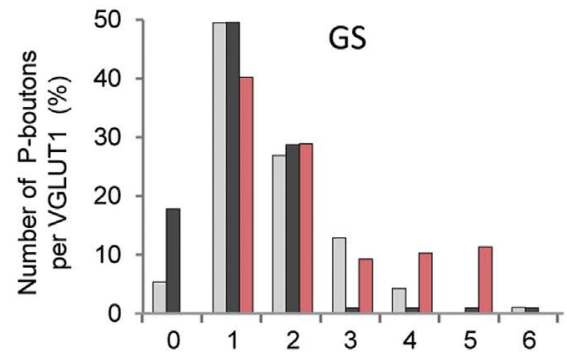

B2

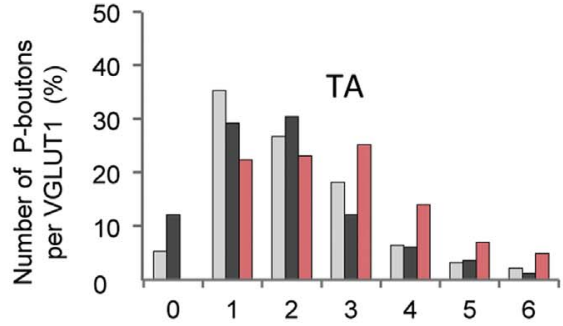

C
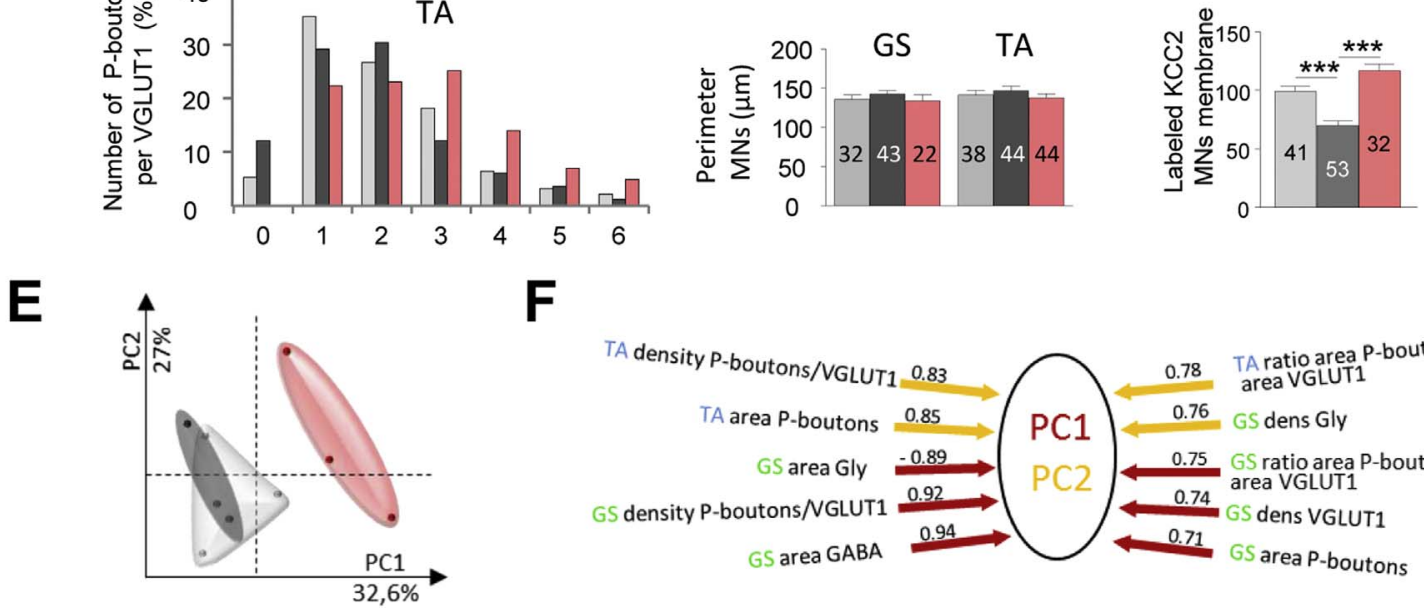

F

Intact

SCT- untrained

SCT-trained

D

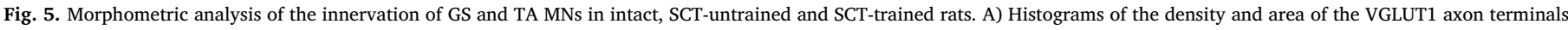

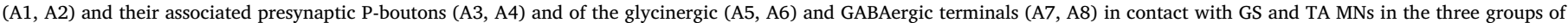

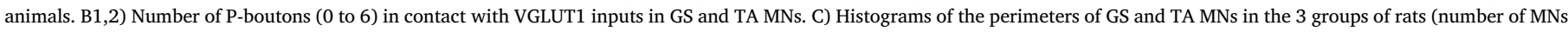

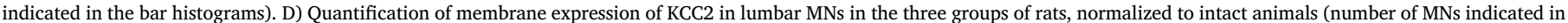

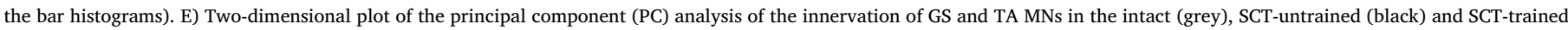

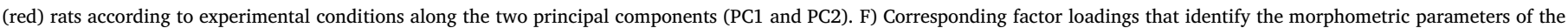

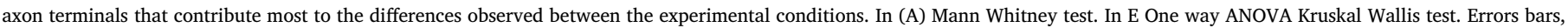
SEM, ${ }^{*} P<0.05 ;{ }^{* *} P<0.01 ; * * * P<0.001$.

\subsection{Reorganization of premotor connectivity to GS MNs in SCT-trained rats}

The "heat maps" of GS premotor INs obtained in the present study in adult intact rats match up to the distribution reported in P15 mice (Tripodi et al., 2011) when using monosynaptically restricted transsynaptic virus. In SCT-untrained rats, there was a trend for impoverishment of the premotor network, whereas in SCT-trained rats, the distribution of premotor INs connected to GS MNs was globally close to the one of intact animals. This is of particular importance in the rostral lumbar segments where central pattern generators (CPGs) are located (Cazalets et al., 1995; Kiehn, 2006), suggesting a preservation of the structural organization of CPGs that monosynaptically relay information from sensory motor afferents to MNs. However, our results highlight remodeling of sub-populations of premotor INs with short 

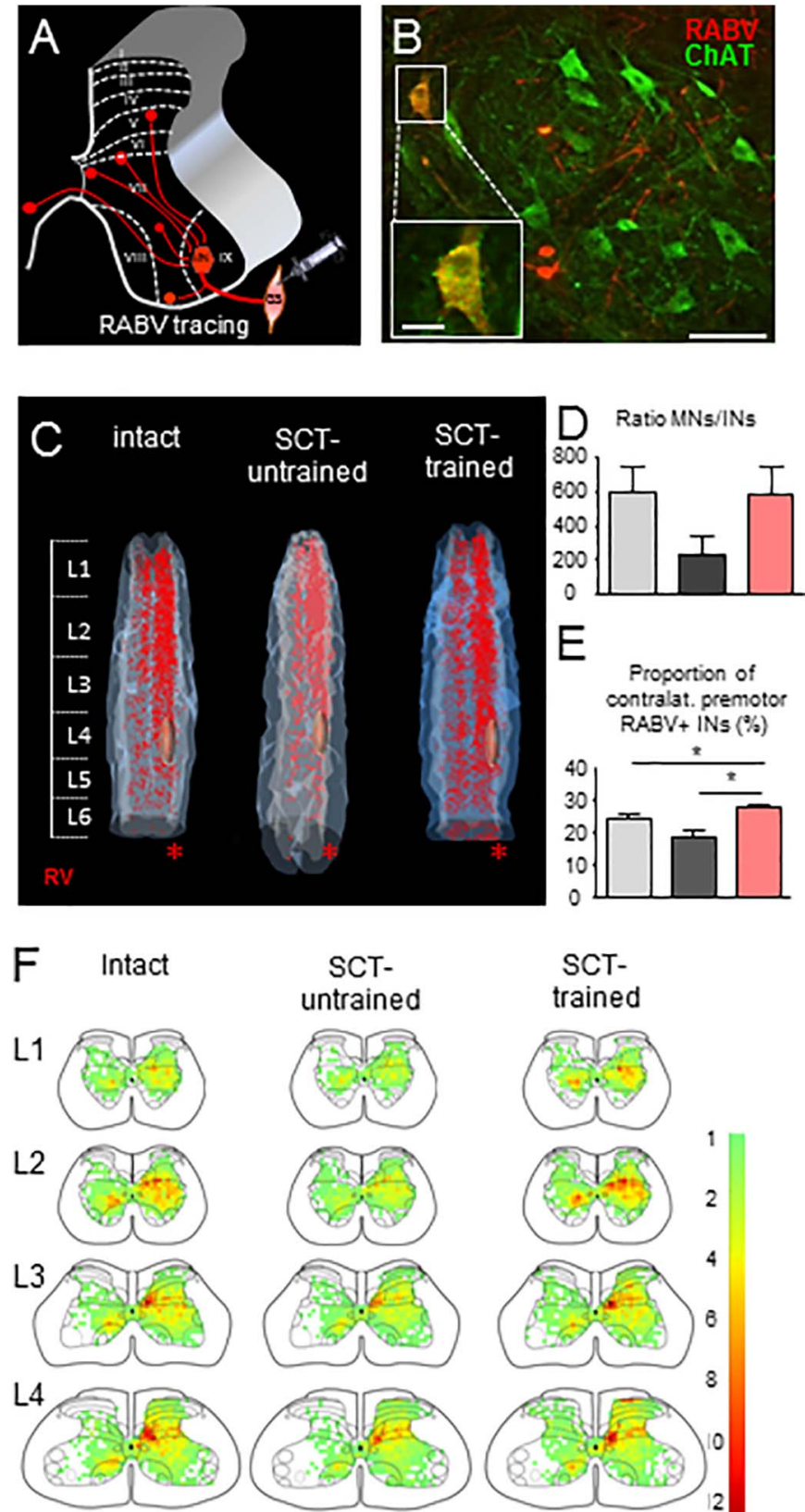

$\mathrm{L} 5$

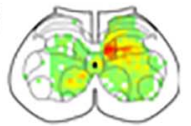

L6
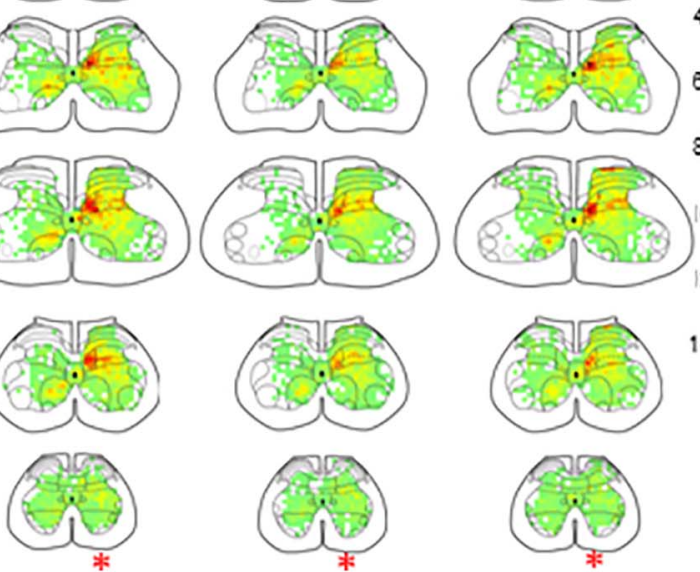

Fig. 6. Transneuronal tracing of premotor network with RABV. A) Diagram illustrating tracing experiments with RABV injected into the right GS muscle. B) GS MN identified by its double immunoreactivity to RABV and ChAT (scale bar: $50 \mu \mathrm{m}$; inset: $25 \mu \mathrm{m}$ ). C) Representative examples of Neurolucida 3D reconstructions of the $\mathrm{RABV}^{+}$-premotor INs distribution (red) in the lumbar segments L1-L6, in intact, SCT-untrained and SCT-trained rats (one out of 12 sections was analyzed). The GS motoneuronal pool is indicated by an oval shape. D) Ratio premotor INs/MNs in intact (grey), SCT (black) and SCT-trained (red) rats. E) Percentage of contralateral premotor INs in the whole lumbar segments the three groups of rats. F) Transverse projections of digital reconstructions (heat maps) of the density of premotor INs (mean of 3 intact rats, 4 SCT rats and 3 SCT-trained rats) in each Rexed lamina of the 6 lumbar segments of intact (left column), SCT-untrained (middle column) and SCT-trained rats (right column). The color scale indicates the number of INs somata in each square. In C and F, the side of injection is marked by an asterisk.
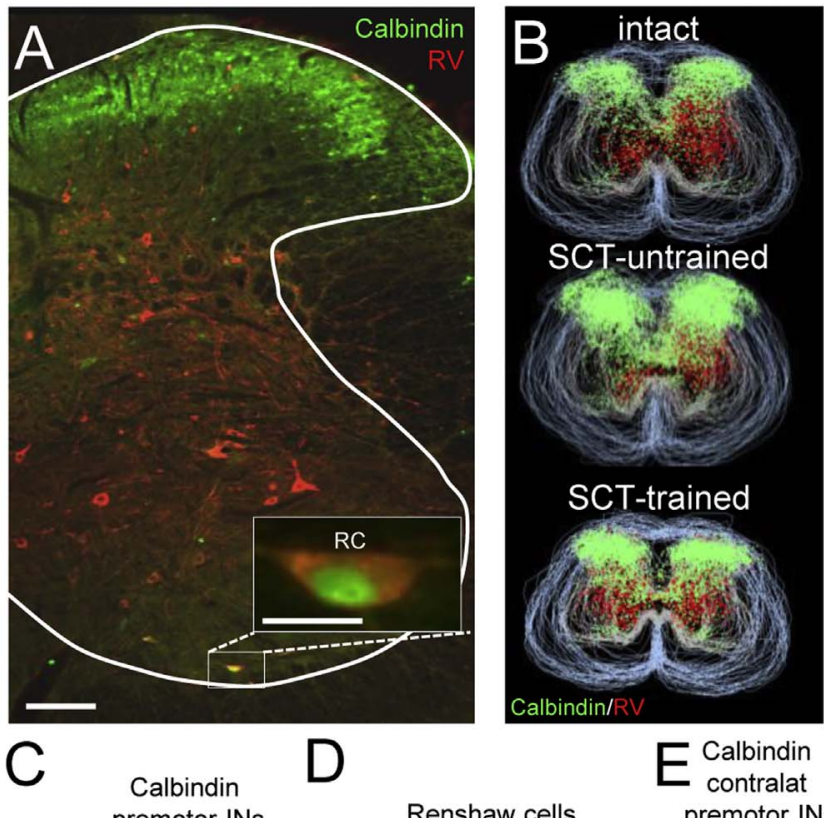

E Calbindin

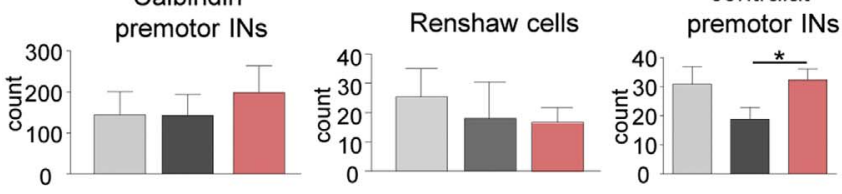

$\square$ Intact $\square$ SCT-untrained $\square$ SCT-trained

F Intact SCT-untrained SCT-trained
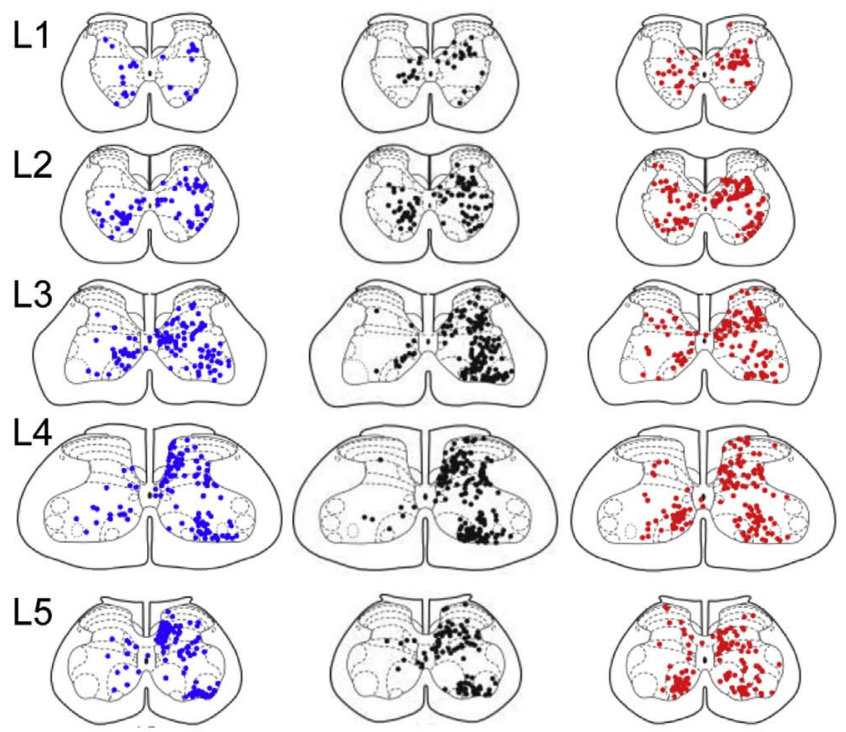

L6
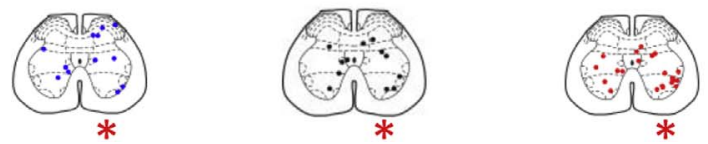

(caption on next page)

connections to GS MNs, in L4-L5 segments. First, we noticed an increase of the number of premotor INs in the ipsilateral superficial laminae of L4-L5 segments. Potential candidates are pacemaker glutamatergic neurons shown to send direct VGLUT2 synapses within motor nuclei at postnatal stages and that serve to facilitate spontaneous muscle contractions (Li et al., 2015). Second, we observed 
Fig. 7. Analysis of calbindin ${ }^{+}$premotor INs in intact, SCT-untrained and SCT-trained rats. A) Example of distribution of calbindin ${ }^{+}$(green) and $\mathrm{RABV}^{+}$premotor (red) INs in L4 lumbar segment in an intact rat. A Renshaw cell (RC in the inset) is characterized by its double immunoreactivity to calbindin and RABV, and its ventral location (scale bar: $50 \mu \mathrm{m}$; enlargement: $20 \mu \mathrm{m}$ ). B) Neurolucida reconstruction in a transverse plan of calbindin $^{+}$(green) and $\mathrm{RABV}^{+}$premotor (red) INs position along the lumbar segments for the three groups of rats. C) Count of double labeled $\mathrm{RABV}^{+} /$calbindin ${ }^{+}$premotor INs in the whole lumbar segments (one out of 12 sections was analyzed) for the three groups of rats. D) count of the RCs connected to GS MNs (RABV-infected) in the whole lumbar segments in each group of animals. E) Count of the contralateral calbindin ${ }^{+} / \mathrm{RABV}^{+}$ premotor INs along the lumbar segments in each group of rats (mean \pm SEM, Mann and Whitney test). F) Transverse projections of digital reconstructions of the calbindin ${ }^{+}$/ $\mathrm{RABV}^{+}$premotor INs in each lumbar segment of intact (left column, blue dots, $n=3$ ), SCT-untrained (middle column, black dots, $n=4$ ) and SCT-trained rats (right column, red dots $n=3$ ). The side of injection is marked by an asterisk.

supernumerary premotor INs in the contralateral lamina V of L4 segment. Such commissural premotor INs have been described in normal adult rats (Puskar and Antal, 1997). Axonal sprouting of lamina V premotor INs onto MNs could be promoted by the potent VGLUT1 innervation observed in this region in SCT-trained rats (Supplementary Fig. 4). Third, there is a decrease in the number of premotor INs located in ipsilateral lamina VII of L4 segment, at the border of the motor nuclei, in both SCT-untrained and SCT-trained animals. This region mainly contains V1-derived premotor INs including Ia INs and RCs (Alvarez et al., 2005), respectively responsible for reciprocal and recurrent inhibition. Our results show that the number of RCs did not significantly change in SCT-trained rats compared to intact animals, and, although the subpopulation of parvalbumin INs was not analyzed in the present study, this suggest that only the population of Ia INs decreased. Such differential preservation could be explained by the fact that Ia INs normally receive potent supraspinal inputs (Hultborn, 2001), while RCs are innervated by only sparse descending afferents (Alvarez and Fyffe, 2007). Therefore, as suggested by Kapitza et al. (2012) the deprivation of supraspinal input after SCT could lead to specific transsynaptic degeneration of some Ia INs, due to withdrawal of trophic support, which would not affect RCs. This suggests that ipsilateral cocontraction of flexor and extensor muscles and reduction of reciprocal inhibition commonly observed in SCI patients (Boorman et al., 1996; Harkema, 2008) could result from a deficit in Ia premotor INs.

\subsection{Functional re-organization of the innervation of lumbar MNs in SCT- trained rats}

Globally, the size and density of all the inputs analyzed in this study (GABA, Glycine, VGLUT1 and P-boutons) similarly changed in GS and TA MNs after training of SCT rats (Fig. 5). These similarities were expected since, on both GS and TA MNs, amplitudes of monosynaptic EPSPs from group Ia afferents in the homonymous muscle and of disynaptic IPSPs from group I afferents in the antagonistic muscle are similar (Khristy et al., 2009). However, different effects were observed when comparing cholinergic inputs on TA and Soleus MNs (Skup et al., 2012). This discrepancy can be explained by the fact that they compared fast flexor (TA) and slow extensor (soleus) rather than fast flexor (TA) and fast extensor (GS) ankle MNs (Khristy et al., 2009). In SCTtrained rats, we found an increase of the size of sensory primary Ia VGLUT1 inputs onto lumbar MNs, which was consistent with the development of an enhanced glutamatergic drive (Cantoria et al., 2011). However, this strengthening might be modulated by the simultaneous increase in the density and size of the presynaptic P-boutons, whose cells of origin are located in the medial part of ipsilateral laminae $\mathrm{V}$ and VI (Hughes et al., 2005). Interestingly, our results show that this region displays increased levels of VGLUT1 innervation, compared to controls (Supplementary Fig. 4). Therefore, we suggest that exercise training could enhance the excitatory VGLUT1 drive both onto lumbar MNs and onto cells of origin of P-boutons, favoring a sprouting of their axonal inputs. In SCT-trained rats, we also observed a significant increase of the density of inhibitory GABAergic and glycinergic inputs, on both GS and TA MNs, but a reduction of their size, which suggest either a sprouting of existing axon terminals, or the presence of inhibitory INs newly connected to MNs. The increase of calbindin ${ }^{+}$premotor INs in the contralateral laminae VII and VIII of L5 segment could be an argument in favor of this latter possibility. Therefore, we suggest that the new rotate stepping pattern adopted by SCT-trained rats, which favors left right alternation of the hindlimbs in the absence of hindlimb/ forelimb coordination, is associated with a strengthening of commissural inhibitory connections to extensor MNs.

\section{Conclusions}

The present results show that combining multisensory motor exercise training and delayed pharmacology can result in recovery of unassisted overground stepping in transected adult rats. The use of this procedure makes it possible to globally maintain the structural organization of the lumbar network associated with adjustments of the innervation of MNs. These remodelings of the circuitry in sub-lesional lumbar segments may contribute to generation of new locomotor patterns.

Supplementary data to this article can be found online at http://dx. doi.org/10.1016/j.expneurol.2017.09.002.

\section{Acknowledgments}

This paper is dedicated to the memory of Laurent Vinay who was a great team leader, a distinguished man. We are grateful to $\mathrm{E}$. Jankowska, and L. Jordan for their invaluable advices and to T.J.H. Ruigrok for revising the manuscript. We thank M. Esclapez for giving us access to her Neurolucida imaging system, D. Blondel, for the gift of the initial batch of CVS- $\mathrm{N}_{2} \mathrm{C}$, F. Jaillet for computing the maps of premotor networks, P. Marino (NS-Repair) for performing the surgery and nursing of the animals, S. Liabeuf for her help in making movies and R. Bras for building the training carrousel. L.K. received a fellowship from the Wings for Life Foundation for Spinal Cord Research (grant FR-027/ 13), K.S. received a grant from the Association Française contre les Myopathies (Grant 13912) and H.B. is supported by Institut National de la Santé et la Recherche Médicale. This research was financed by the ANR-2010-BLAN-1407-01. The authors declare no competing financial interests.

\section{References}

Alluin, O., Delivet-Mongrain, H., Rossignol, S., 2015. Inducing hindlimb locomotor recovery in adult rat after complete thoracic spinal cord section using repeated treadmill training with perineal stimulation only. J. Neurophysiol. 114, 1931-1946. Alvarez, F.J., Fyffe, R.E., 2007. The continuing case for the Renshaw cell. J. Physiol. 584, $31-45$.

Alvarez, F.J., Jonas, P.C., Sapir, T., Hartley, R., Berrocal, M.C., Geiman, E.J., Todd, A.J., Goulding, M., 2005. Postnatal phenotype and localization of spinal cord V1 derived interneurons. J. Comp. Neurol. 493, 177-192.

Antri, M., Orsal, D., Barthe, J.Y., 2002. Locomotor recovery in the chronic spinal rat: effects of long-term treatment with a 5-HT2 agonist. Eur. J. Neurosci. 16, 467-476. Basso, D.M., Beattie, M.S., Bresnahan, J.C., 1995. A sensitive and reliable locomotor rating scale for open field testing in rats. J. Neurotrauma 12, 1-21.

Battistuzzo, C.R., Callister, R.J., Callister, R., Galea, M.P., 2012. A systematic review of exercise training to promote locomotor recovery in animal models of spinal cord injury. J. Neurotrauma 29, 1600-1613.

Boorman, G.I., Lee, R.G., Becker, W.J., Windhorst, U.R., 1996. Impaired "natural reciprocal inhibition" in patients with spasticity due to incomplete spinal cord injury. Electroencephalogr. Clin. Neurophysiol. 101, 84-92.

Bos, R., Sadlaoud, K., Boulenguez, P., Buttigieg, D., Liabeuf, S., Brocard, C., Haase, G., Bras, H., Vinay, L., 2013. Activation of 5-HT2A receptors upregulates the function of the neuronal K-Cl cotransporter KCC2. Proc. Natl. Acad. Sci. U. S. A. 110, 348-353.

Boulenguez, P., Liabeuf, S., Bos, R., Bras, H., Jean-Xavier, C., Brocard, C., Stil, A., Darbon, P., Cattaert, D., Delpire, E., Marsala, M., Vinay, L., 2010. Down-regulation of the potassium-chloride cotransporter KCC2 contributes to spasticity after spinal cord injury. Nat. Med. 16, 302-307.

Brumovsky, P., Watanabe, M., Hokfelt, T., 2007. Expression of the vesicular glutamate transporters- 1 and -2 in adult mouse dorsal root ganglia and spinal cord and their regulation by nerve injury. Neuroscience $147,469-490$. 
Burke, D.A., Magnuson, D.S., Nunn, C.D., Fentress, K.G., Wilson, M.L., Shum-Siu, A.H., Moore, M.C., Turner, L.E., King, W.W., Onifer, S.M., 2007. Use of environmentally enriched housing for rats with spinal cord injury: the need for standardization. J. Am. Assoc. Lab. Anim. Sci. 46, 34-41.

Cantoria, M.J., See, P.A., Singh, H., de Leon, R.D., 2011. Adaptations in glutamate and glycine content within the lumbar spinal cord are associated with the generation of novel gait patterns in rats following neonatal spinal cord transection. J. Neurosci. 31, 18598-18605.

Carr, P.A., Alvarez, F.J., Leman, E.A., Fyffe, R.E., 1998. Calbindin D28k expression in immunohistochemically identified Renshaw cells. Neuroreport 9, 2657-2661. Cazalets, J.R., Borde, M., Clarac, F., 1995. Localization and organization of the central 4951. pattern generator for hindlimb locomotion in newborn rat. J. Neurosci. 15, 4943-

Cha, J., Heng, C., Reinkensmeyer, D.J., Roy, R.R., Edgerton, V.R., de Leon, R.D., 2007. Locomotor ability in spinal rats is dependent on the amount of activity imposed on the hindlimbs during treadmill training. J. Neurotrauma 24, 1000-1012.

Cheng, H., Almstrom, S., Gimenez-Llort, L., Chang, R., Ove, O.S., Hoffer, B., Olson, L., 1997. Gait analysis of adult paraplegic rats after spinal cord repair. Exp. Neurol. 148, 544-557.

Cote, M.P., Detloff, M.R., Wade Jr., R.E., Lemay, M.A., Houle, J.D., 2012. Plasticity in ascending long propriospinal and descending supraspinal pathways in chronic cervical spinal cord injured rats. Front. Physiol. 3, 330. http://dx.doi.org/10.3389/ fphys.2012.00330. (eCollection@2012., 330).

Cote, M.P., Gandhi, S., Zambrotta, M., Houle, J.D., 2014. Exercise modulates chloride homeostasis after spinal cord injury. J. Neurosci. 34, 8976-8987.

Coulon, P., Bras, H., Vinay, L., 2011. Characterization of last-order premotor interneurons by transneuronal tracing with rabies virus in the neonatal mouse spinal cord. J. Comp. Neurol. 519, 3470-3487.

Courtine, G., Gerasimenko, Y., van Den, B.R., Yew, A., Musienko, P., Zhong, H., Song, B., Ao, Y., Ichiyama, R.M., Lavrov, I., Roy, R.R., Sofroniew, M.V., Edgerton, V.R., 2009. Transformation of nonfunctional spinal circuits into functional states after the loss of brain input. Nat. Neurosci. 12, 1333-1342.

Doyon, N., Vinay, L., Prescott, S.A., De, K.Y., 2016. Chloride regulation: a dynamic equilibrium crucial for synaptic inhibition. Neuron $89,1157-1172$

Druga, R., 2009. Neocortical inhibitory system. Folia Biol. (Praha). 55, 201-217.

Dugan, E.A., Shumsky, J.S., 2015. A combination therapy of neural and glial restricted precursor cells and chronic quipazine treatment paired with passive cycling promotes quipazine-induced stepping in adult spinalized rats. J. Spinal Cord. Med. 38, 792-804.

Edgerton, V.R., Courtine, G., Gerasimenko, Y.P., Lavrov, I., Ichiyama, R.M., Fong, A.J., Cai, L.L., Otoshi, C.K., Tillakaratne, N.J., Burdick, J.W., Roy, R.R., 2008. Training

locomotor networks. Brain Res. Rev. 57, 241-254.

Esclapez, M., Tillakaratne, N.J., Kaufman, D.L., Tobin, A.J., Houser, C.R., 1994 Comparative localization of two forms of glutamic acid decarboxylase and their mRNAs in rat brain supports the concept of functional differences between the forms. J. Neurosci. 14, 1834-1855.

Feraboli-Lohnherr, D., Barthe, J.Y., Orsal, D., 1999. Serotonin-induced activation of the network for locomotion in adult spinal rats. J. Neurosci. Res. 55, 87-98.

Field, A., Miles, J., Field, Z., 2012. Discovering Statistics Using R. SAGE Publications, London UK.

Fong, A.J., Cai, L.L., Otoshi, C.K., Reinkensmeyer, D.J., Burdick, J.W., Roy, R.R., Edgerton, V.R., 2005. Spinal cord-transected mice learn to step in response to qui-

pazine treatment and robotic training. J. Neurosci. 25, 11738-11747.

Fouquet, B., Ghosn, J., Quertainmont, Y., Salmon, D., Rioux, C., Duvivier, C., Delfraissy, J.F., Misrahi, M., 2015. Identification of variants of hepatitis C virus (HCV) entry factors in patients highly exposed to HCV but remaining uninfected: an ANRS casecontrol study. PLoS One 10, e0142698.

Gillis, G.B., Biewener, A.A., 2001. Hindlimb muscle function in relation to speed and gait: in vivo patterns of strain and activation in a hip and knee extensor of the rat (Rattus norvegicus). J. Exp. Biol. 204, 2717-2731.

Guertin, P.A., 2004. Synergistic activation of the central pattern generator for locomotion by l-beta-3,4-dihydroxyphenylalanine and quipazine in adult paraplegic mice. Neurosci. Lett. 358, 71-74.

Hamers, F.P., Lankhorst, A.J., van Laar, T.J., Veldhuis, W.B., Gispen, W.H., 2001. Automated quantitative gait analysis during overground locomotion in the rat: its application to spinal cord contusion and transection injuries. J. Neurotrauma 18 , 187-201.

Harkema, S.J., 2008. Plasticity of interneuronal networks of the functionally isolated human spinal cord. Brain Res. Rev. 57, 255-264.

Harkema, S.J., Hillyer, J., Schmidt-Read, M., Ardolino, E., Sisto, S.A., Behrman, A.L., 2012. Locomotor training: as a treatment of spinal cord injury and in the progression of neurologic rehabilitation. Arch. Phys. Med. Rehabil. 93, 1588-1597.

Holstege, J.C., Kuypers, H.G., 1987. Brainstem projections to lumbar motoneurons in rat-I. An ultrastructural study using autoradiography and the combination of autoradiography and horseradish peroxidase histochemistry. Neuroscience 21, 345-367.

Hughes, D.I., Mackie, M., Nagy, G.G., Riddell, J.S., Maxwell, D.J., Szabo, G., Erdelyi, F., Veress, G., Szucs, P., Antal, M., Todd, A.J., 2005. P boutons in lamina IX of the rodent spinal cord express high levels of glutamic acid decarboxylase-65 and originate from cells in deep medial dorsal horn. Proc. Natl. Acad. Sci. U. S. A. 102, 9038-9043.

Hultborn, H., 2001. State-dependent modulation of sensory feedback. J. Physiol. 533, 5-13.

Ichiyama, R.M., Broman, J., Roy, R.R., Zhong, H., Edgerton, V.R., Havton, L.A., 2011. Locomotor training maintains normal inhibitory influence on both alpha- and gamma-motoneurons after neonatal spinal cord transection. J. Neurosci. 31, 26-33.

Kapitza, S., Zorner, B., Weinmann, O., Bolliger, M., Filli, L., Dietz, V., Schwab, M.E., 2012.
Tail spasms in rat spinal cord injury: changes in interneuronal connectivity. Exp. Neurol. 236, 179-189.

Kasumacic, N., Lambert, F.M., Coulon, P., Bras, H., Vinay, L., Perreault, M.C., Glover, J.C., 2015. Segmental organization of vestibulospinal inputs to spinal interneurons mediating crossed activation of thoracolumbar motoneurons in the neonatal mouse. J. Neurosci. 35, 8158-8169.

Khalki, L., Ba, M.S., Sokar, Z., Bennis, M., Vinay, L., Bras, H., Viemari, J.C., 2013. Prenatal exposure to fenugreek impairs sensorimotor development and the operation of spinal cord networks in mice. PLoS One 8, e80013.

Khristy, W., Ali, N.J., Bravo, A.B., de Leon, R., Roy, R.R., Zhong, H., London, N.J., Edgerton, V.R., Tillakaratne, N.J., 2009. Changes in GABA(A) receptor subunit gamma 2 in extensor and flexor motoneurons and astrocytes after spinal cord transection and motor training. Brain Res. 1273, 9-17.

Kiehn, O., 2006. Locomotor circuits in the mammalian spinal cord. Annu. Rev. Neurosci. 29, 279-306.

Kitzman, P., 2007. VGLUT1 and GLYT2 labeling of sacrocaudal motoneurons in the spinal cord injured spastic rat. Exp. Neurol. 204, 195-204.

Lankhorst, A.J., ter Laak, M.P., van Laar, T.J., van Meeteren, N.L., de Groot, J.C., Schrama, L.H., Hamers, F.P., Gispen, W.H., 2001. Effects of enriched housing on functional recovery after spinal cord contusive injury in the adult rat. J. Neurotrauma 18, 203-215

de Leon, R.D., Acosta, C.N., 2006. Effect of robotic-assisted treadmill training and chronic quipazine treatment on hindlimb stepping in spinally transected rats. J. Neurotrauma

23, 1147-1163.

de Leon, R.D., Hodgson, J.A., Roy, R.R., Edgerton, V.R., 1998. Full weight-bearing hindlimb standing following stand training in the adult spinal cat. J. Neurophysiol. 80, 83-91.

Li, J., Kritzer, E., Ford, N.C., Arbabi, S., Baccei, M.L., 2015. Connectivity of pacemaker neurons in the neonatal rat superficial dorsal horn. J. Comp. Neurol. 523, 1038-1053. Lorenzo, L.E., Magnussen, C., Bailey, A.L., St, L.M., De, K.Y., Ribeiro-da-Silva, A., 2014 Spatial and temporal pattern of changes in the number of GAD65-immunoreactive inhibitory terminals in the rat superficial dorsal horn following peripheral nerve injury. Mol. Pain 10, 57. http://dx.doi.org/10.1186/1744-8069-10-57. (57-10).

Martinez, M., Brezun, J.M., Zennou-Azogui, Y., Baril, N., Xerri, C., 2009. Sensorimotor training promotes functional recovery and somatosensory cortical map reactivation

following cervical spinal cord injury. Eur. J. Neurosci. 30, 2356-2367.

van Meeteren, N.L., Eggers, R., Lankhorst, A.J., Gispen, W.H., Hamers, F.P., 2003 Locomotor recovery after spinal cord contusion injury in rats is improved by spon-taneous exercise. J. Neurotrauma 20, 1029-1037.

Miya, D., Giszter, S., Mori, F., Adipudi, V., Tessler, A., Murray, M., 1997. Fetal transplants alter the development of function after spinal cord transection in newborn rats. J.

Neurosci. 17, 4856-4872.

Molander, C., Xu, Q., Grant, G., 1984. The cytoarchitectonic organization of the spinal cord in the rat. I. The lower thoracic and lumbosacral cord. J. Comp. Neurol. 230, 133-141.

Moshonkina, T.R., Gilerovich, E.G., Fedorova, E.A., Avelev, V.D., Gerasimenko, Y.P., Otellin, V.A., 2004. Morphofunctional basis for recovery of locomotor movements in rats with completely crossed spinal cord. Bull. Exp. Biol. Med. 138, 198-201.

Musienko, P., van Den, B.R., Marzendorfer, O., Roy, R.R., Gerasimenko, Y., Edgerton, V.R., Courtine, G., 2011. Controlling specific locomotor behaviors through multidimensional monoaminergic modulation of spinal circuitries. J. Neurosci. 31 , 9264-9278.

Nothias, J.M., Mitsui, T., Shumsky, J.S., Fischer, I., Antonacci, M.D., Murray, M., 2005. Combined effects of neurotrophin secreting transplants, exercise, and serotonergic drug challenge improve function in spinal rats. Neurorehabil. Neural Repair 19, 296-312.

Orsal, D., Barthe, J.Y., Antri, M., Feraboli-Lohnherr, D., Yakovleff, A., Ribotta, M., Privat A., Provencher, J., Rossignol, S., 2002. Locomotor recovery in chronic spinal rat: long-term pharmacological treatment or transplantation of embryonic neurons? Prog. Brain Res. 137 (213-30), 213-230.

Puskar, Z., Antal, M., 1997. Localization of last-order premotor interneurons in the lumbar spinal cord of rats. J. Comp. Neurol. 389, 377-389.

Rossignol, S., 2006. Plasticity of connections underlying locomotor recovery after central and/or peripheral lesions in the adult mammals. Philos. Trans. R. Soc. Lond. Ser. B

Biol. Sci. 361, 1647-1671.

Rossignol, S., Frigon, A., 2011. Recovery of locomotion after spinal cord injury: some facts and mechanisms. Annu. Rev. Neurosci. 34, 413-440.

Roy, R.R., Harkema, S.J., Edgerton, V.R., 2012. Basic concepts of activity-based interventions for improved recovery of motor function after spinal cord injury. Arch. Phys.

Med. Rehabil. 93, 1487-1497.

Ruigrok, T.J., Pijpers, A., Goedknegt-Sabel, E., Coulon, P., 2008. Multiple cerebellar zones are involved in the control of individual muscles: a retrograde transneuronal tracing study with rabies virus in the rat. Eur. J. Neurosci. 28, 181-200.

Sadlaoud, K., Tazerart, S., Brocard, C., Jean-Xavier, C., Portalier, P., Brocard, F., Vinay, L., Bras, H., 2010. Differential plasticity of the GABAergic and glycinergic synaptic transmission to rat lumbar motoneurons after spinal cord injury. J. Neurosci. 30 3358-3369.

Skup, M., Gajewska-Wozniak, O., Grygielewicz, P., Mankovskaya, T., Czarkowska-Bauch, J., 2012. Different effects of spinalization and locomotor training of spinal animals on cholinergic innervation of the soleus and tibialis anterior motoneurons. Eur. J. Neurosci. 36, 2679-2688.

Slawinska, U., Majczynski, H., Dai, Y., Jordan, L.M., 2012. The upright posture improves plantar stepping and alters responses to serotonergic drugs in spinal rats. J. Physiol.

$590,1721-1736$

Tillakaratne, N.J., Mouria, M., Ziv, N.B., Roy, R.R., Edgerton, V.R., Tobin, A.J., 2000. Increased expression of glutamate decarboxylase (GAD(67)) in feline lumbar spinal 
cord after complete thoracic spinal cord transection. J. Neurosci. Res. 60, 219-230. Tillakaratne, N.J., de Leon, R.D., Hoang, T.X., Roy, R.R., Edgerton, V.R., Tobin, A.J., 2002. Use-dependent modulation of inhibitory capacity in the feline lumbar spinal cord. J. Neurosci. 22, 3130-3143.

Tillakaratne, N.J., Guu, J.J., de Leon, R.D., Bigbee, A.J., London, N.J., Zhong, H., Ziegler, M.D., Joynes, R.L., Roy, R.R., Edgerton, V.R., 2010. Functional recovery of stepping in rats after a complete neonatal spinal cord transection is not due to regrowth across the lesion site. Neuroscience 166, 23-33.

Timoszyk, W.K., Nessler, J.A., Acosta, C., Roy, R.R., Edgerton, V.R., Reinkensmeyer, D.J., de Leon, R., 2005. Hindlimb loading determines stepping quantity and quality following spinal cord transection. Brain Res. 1050, 180-189.

Todd, A.J., Hughes, D.I., Polgar, E., Nagy, G.G., Mackie, M., Ottersen, O.P., Maxwell, D.J., 2003. The expression of vesicular glutamate transporters VGLUT1 and VGLUT2 in neurochemically defined axonal populations in the rat spinal cord with emphasis on the dorsal horn. Eur. J. Neurosci. 17, 13-27.

Tripodi, M., Stepien, A.E., Arber, S., 2011. Motor antagonism exposed by spatial segregation and timing of neurogenesis. Nature 479, 61-66.

Wang, H., Liu, N.K., Zhang, Y.P., Deng, L., Lu, Q.B., Shields, C.B., Walker, M.J., Li, J., Xu, X.M., 2015. Treadmill training induced lumbar motoneuron dendritic plasticity and behavior recovery in adult rats after a thoracic contusive spinal cord injury. Exp.

Neurol. 271, 368-378. http://dx.doi.org/10.1016/j.expneurol.2015.07.004. (Epub@ 2015 Jul 9., 368-378).

Weber, E.D., Stelzner, D.J., 1977. Behavioral effects of spinal cord transection in the developing rat. Brain Res. 125, 241-255.

Zhang, Y., Ji, S.R., Wu, C.Y., Fan, X.H., Zhou, H.J., Liu, G.L., 2007. Observation of locomotor functional recovery in adult complete spinal rats with BWSTT using semiquantitative and qualitative methods. Spinal Cord 45, 496-501. 